\title{
Multiplicity of Positive Solutions for Semilinear Elliptic Systems
}

\author{
Tsing-San Hsu \\ Department of Natural Sciences in the Center for General Education, Chang Gung University, Tao-Yuan 333, Taiwan
}

Correspondence should be addressed to Tsing-San Hsu; tshsu@mail.cgu.edu.tw

Received 15 November 2012; Accepted 5 March 2013

Academic Editor: Gabriella Tarantello

Copyright (C) 2013 Tsing-San Hsu. This is an open access article distributed under the Creative Commons Attribution License, which permits unrestricted use, distribution, and reproduction in any medium, provided the original work is properly cited.

We study the effect of the coefficient $h(x)$ of the critical nonlinearity on the number of positive solutions for semilinear elliptic systems. Under suitable assumptions for $f(x), g(x)$, and $h(x)$, we should prove that for sufficiently small $\lambda, \mu>0$, there are at least $k+1$ positive solutions of the semilinear elliptic systems $-\Delta u=\lambda f(x)|u|^{q-2} u+(\alpha /(\alpha+\beta)) h(x)|u|^{\alpha-2} u|v|^{\beta},-\Delta v=\mu g(x)|v|^{q-2} v+$ $(\beta /(\alpha+\beta)) h(x)|u|^{\alpha}|v|^{\beta-2} v$, where $0 \in \Omega \subset \mathbb{R}^{N}$ is a bounded domain, $\alpha>1, \beta>1$, and $N /(N-2)<q<2<\alpha+\beta=2^{*}$ for $N>4$.

\section{Introduction and Main Results}

For $N \geq 3, \alpha>1, \beta>1$, and $1 \leq q<2<\alpha+\beta=2^{*}=$ $2 N /(N-2)$, consider the semilinear elliptic systems

$$
\begin{cases}-\Delta u=\lambda f(x)|u|^{q-2} u+\frac{\alpha}{\alpha+\beta} h(x)|u|^{\alpha-2} u|v|^{\beta} & \text { in } \Omega, \\ -\Delta v=\mu g(x)|v|^{q-2} v+\frac{\beta}{\alpha+\beta} h(x)|u|^{\alpha}|v|^{\beta-2} v & \text { in } \Omega, \\ u=v=0 & \text { on } \partial \Omega,\end{cases}
$$

where $\lambda, \mu>0, \Omega \subset \mathbb{R}^{N}$ is a bounded domain with smooth boundary $\partial \Omega$.

Let $f, g$, and $h$ satisfy the following conditions.

(H1) $f, g$, and $h$ are positive continuous functions in $\bar{\Omega}$ and $\max _{x \in \bar{\Omega}} h(x)=1$.

(H2) There exist $k$ points $a_{1}, a_{2}, \ldots, a_{k} \in \Omega$ and some $\sigma \geq$ $N-2$ such that $h\left(a_{i}\right)$ are strict maxima and satisfy

$$
h\left(a_{i}\right)=\max _{x \in \bar{\Omega}} h(x)=1 \quad \forall 1 \leq i \leq k
$$

and $h(x)=h\left(a_{i}\right)+O\left(\left|x-a_{i}\right|^{\sigma}\right)$ as $x \rightarrow a_{i}$ uniformly in $i$.

Recent studies [1-10] have investigated the elliptic systems with subcritical or critical exponents and have proved the existence of a ground state solution or the existence of at least two positive solutions for these problems. For the case of
$N>4, \alpha>1, \beta>1$, and $2<q<\alpha+\beta=2^{*}=2 N /(N-2)$, Lin [11] constructs the $k$ compact Palais-Smale sequences that are suitably localized in correspondence of $k$ maximum points of $h$. Under assumptions (H1)-(H2), she has showed that there are at least $k$ positive solutions of the problem $\left(P_{\lambda, \mu}\right)$ for sufficiently small $\lambda, \mu>0$. In this paper, we study the problem $\left(P_{\lambda, \mu}\right)$ and complement the results of [11] to the case $1 \leq q<2$. Under assumptions (H1)-(H2), we should prove that there exist at least $k+1$ positive solutions of the problem $\left(P_{\lambda, \mu}\right)$ for sufficiently small $\lambda, \mu>0$.

Let $E=H_{0}^{1}(\Omega) \times H_{0}^{1}(\Omega)$ be the space with the standard norm

$$
\|(u, v)\|_{E}=\left(\int_{\Omega}\left(|\nabla u|^{2}+|\nabla v|^{2}\right) d x\right)^{1 / 2} .
$$

Associated with the problem $\left(P_{\lambda, \mu}\right)$, we consider the $C^{1}$-functional $I_{\lambda, \mu}$, for $(u, v) \in E$,

$$
\begin{aligned}
I_{\lambda, \mu}(u, v)= & \frac{1}{2}\|(u, v)\|_{E}^{2} \\
& -\frac{1}{q} \int_{\Omega}\left(\lambda f(x)|u|^{q}+\mu g(x)|v|^{q}\right) d x \\
& -\frac{1}{2^{*}} \int_{\Omega} h(x)|u|^{\alpha}|v|^{\beta} d x .
\end{aligned}
$$


The weak solution $(u, v) \in E$ of the problem $\left(P_{\lambda, \mu}\right)$ is the critical point of the functional $I_{\lambda, \mu}$; that is, $(u, v) \in E$ satisfies

$$
\begin{aligned}
\int_{\Omega}\left(\nabla u \nabla \varphi_{1}+\nabla v \nabla \varphi_{2}\right) d x-\lambda \int_{\Omega} f(x)|u|^{q-2} u \varphi_{1} d x \\
-\mu \int_{\Omega} g(x)|v|^{q-2} v \varphi_{2} d x-\frac{\alpha}{2^{*}} \int_{\Omega} h(x)|u|^{\alpha-2} u|v|^{\beta} \varphi_{1} d x \\
\quad-\frac{\beta}{2^{*}} \int_{\Omega} h(x)|u|^{\alpha}|v|^{\beta-2} v \varphi_{2} d x=0
\end{aligned}
$$

for any $\left(\varphi_{1}, \varphi_{2}\right) \in E$.

Let $D^{1,2}\left(\mathbb{R}^{N}\right)=\left\{u \in L^{2^{*}}\left(\mathbb{R}^{N}\right) \mid \nabla u \in\left(L^{2}\left(\mathbb{R}^{N}\right)\right)^{N}\right\}$ with the norm $\|u\|^{2}=\int_{\mathbb{R}^{N}}|\nabla u|^{2} d x$, and let $S$ be the best Sobolev constant defined by

$$
\begin{gathered}
S=\inf _{u \in D^{1,2}\left(\mathbb{R}^{N}\right) \backslash\{0\}} \frac{\int_{\mathbb{R}^{N}}|\nabla u|^{2} d x}{\left(\int_{\mathbb{R}^{N}}|u|^{2^{*}} d x\right)^{2 / 2^{*}}} \\
\left(=\inf _{u \in H_{0}^{1}(\Omega) \backslash\{0\}} \frac{\int_{\Omega}|\nabla u|^{2} d x}{\left(\int_{\Omega}|u|^{2^{*}} d x\right)^{2 / 2^{*}}}\right)>0,
\end{gathered}
$$

and let

$$
S_{\alpha, \beta}=\inf _{u, v \in H_{0}^{1}(\Omega) \backslash\{0\}} \frac{\int_{\Omega}\left(|\nabla u|^{2}+|\nabla v|^{2}\right) d x}{\left(\int_{\Omega}|u|^{\alpha}|v|^{\beta} d x\right)^{2 /(\alpha+\beta)}} ;
$$

then, by [1, Theorem 5], we have

$$
S_{\alpha, \beta}=\left(\left(\frac{\alpha}{\beta}\right)^{\beta /(\alpha+\beta)}+\left(\frac{\beta}{\alpha}\right)^{\alpha /(\alpha+\beta)}\right) S,
$$

where $\alpha+\beta=2^{*}$.

Set

$$
\begin{aligned}
\Lambda_{1}= & \left(\frac{2-q}{2^{*}-q}\right)^{2 /\left(2^{*}-2\right)}\left(\frac{\left(2^{*}-q\right) \gamma_{\infty}}{2^{*}-2}|\Omega|^{\left(2^{*}-q\right) / 2^{*}}\right)^{-2 /(2-q)} \\
& \times S^{N / 2+q /(2-q)}>0,
\end{aligned}
$$

where $\gamma_{\infty}=\max \left\{|f|_{L^{\infty}(\Omega)},|g|_{L^{\infty}(\Omega)}\right\}$.

The main results of this paper are given as follows.

Theorem 1. Assume that (H1) holds. If $\lambda, \mu>0$ satisfy $0<$ $\lambda^{2 /(2-q)}+\mu^{2 /(2-q)}<\Lambda_{1}$, then there exists at least one positive ground state solution of the problem $\left(P_{\lambda, \mu}\right)$.

Theorem 2. Under the assumptions (H1)-(H2), and N/(N$2)<q<2$ and $N>4$, there exists a positive number $\Lambda^{*} \epsilon$ $\left(0, \Lambda_{1}\right)$ such that for $\lambda, \mu>0$ and $\lambda^{2 /(2-q)}+\mu^{2 /(2-q)}<\Lambda^{*}$, the problem $\left(P_{\lambda, \mu}\right)$ has $k+1$ positive solutions.

This paper is organized as follows. In Section 2, we consider the Nehari manifold

$$
\mathcal{N}_{\lambda, \mu}=\left\{(u, v) \in E \backslash\{0\} \mid\left\langle I_{\lambda, \mu}^{\prime}(u, v),(u, v)\right\rangle=0\right\},
$$

where

$$
\begin{aligned}
\left\langle I_{\lambda, \mu}^{\prime}\right. & (u, v),(u, v)\rangle \\
= & \|(u, v)\|_{E}^{2}-\int_{\Omega}\left(\lambda f(x)|u|^{q}+\mu g(x)|v|^{q}\right) d x \\
& \quad-\int_{\Omega} h(x)|u|^{\alpha}|v|^{\beta} d x .
\end{aligned}
$$

Note that $\mathcal{N}_{\lambda, \mu}$ contains all nontrivial weak solution of the problem $\left(P_{\lambda, \mu}\right)$. Using the argument of Tarantello [12, 13], we split $\mathcal{N}_{\lambda, \mu}$ into two parts $\mathcal{N}_{\lambda, \mu}^{+}$and $\mathcal{N}_{\lambda, \mu}^{-}$for $0<$ $\lambda^{2 /(2-q)}+\mu^{2 /(2-q)}<\Lambda_{1}$. In Section 3, we prove Theorem 1 . In Section 4, since $I_{\lambda, \mu}$ satisfies the (PS) $)_{\gamma}$-condition for $\gamma \in$ $\left(-\infty,(1 / N)\left(S_{\alpha, \beta}\right)^{N / 2}-C_{0}\left(\lambda^{2 /(2-q)}+\mu^{2 /(2-q)}\right)\right)$, for sufficiently small $\lambda$, $\mu$, and some restriction on $q$ and $N$, we construct the $k$ compact Palais-Smale sequences which are suitably localized in correspondence with the $k$ maximum points of $h$ and which converge to distinct solutions of the problem $\left(P_{\lambda, \mu}\right)$ belonging to $\mathcal{N}_{\lambda, \mu}^{-}$. Hence, we prove Theorem 2 (one is the ground state solution belonging to $\mathcal{N}_{\lambda, \mu}^{+}$and the others are in $\mathcal{N}_{\lambda, \mu}^{-}$).

\section{Nehari Manifold}

Throughout this paper, $(H 1)$ will be assumed. First, we give some notations.

Notations. We make use of the following notations.

$L^{p}(\Omega), 1 \leq p \leq \infty$, denote Lebesgue spaces; the norm $L^{p}$ is denoted by $|\cdot|_{L^{p}(\Omega)}$ for $1 \leq p \leq \infty$.

$E=\left[H_{0}^{1}(\Omega)\right]^{2}$, endowed with norm $\|z\|_{E}^{2}=$ $\|(u, v)\|_{E}^{2}=|\nabla u|_{2}^{2}+|\nabla v|_{2}^{2}$.

The dual space of a Banach space $E$ will be denoted by $E^{-1}$.

$|z|=|(u, v)|=(|u|,|v|)$ and $t z=t(u, v)=(t u, t v)$ for all $z \in E$ and $t \in \mathbb{R}$.

$z=(u, v)$ is said to be nonnegative in $\Omega$ if $u \geq 0$ and $v \geq 0$ in $\Omega$.

$z=(u, v)$ is said to be positive in $\Omega$ if $u>0$ and $v>0$ in $\Omega$.

$|\Omega|$ is the Lebesgue measure of $\Omega$.

$B_{r}(a)=\left\{x \in \mathbb{R}^{N}|| x-a \mid<r\right\}$ is a ball in $\mathbb{R}^{N}$.

$O\left(\varepsilon^{t}\right)$ denotes $\left|O\left(\varepsilon^{t}\right)\right| / \varepsilon^{t} \leq C$ as $\varepsilon \rightarrow 0$ for $t \geq 0$.

$\mathrm{O}_{1}\left(\varepsilon^{t}\right)$ means that there exist the constants $C_{1}, C_{2}>0$ such that $C_{1} \varepsilon^{t} \leq O_{1}\left(\varepsilon^{t}\right) \leq C_{2} \varepsilon^{t}$ as $\varepsilon$ is small.

$o_{n}(1)$ denotes $o_{n}(1) \rightarrow 0$ as $n \rightarrow \infty$.

$\gamma_{\infty}=\max \left\{|f|_{L^{\infty}(\Omega)},|g|_{L^{\infty}(\Omega)}\right\}$.

$C, C_{i}$ will denote various positive constants, the exact values of which are not important. 
Let $K_{\lambda, \mu}: E \rightarrow \mathbb{R}$ be the functional defined by

$K_{\lambda, \mu}(z)=\int_{\Omega}\left(\lambda f(x)|u|^{q}+\mu g(x)|v|^{q}\right) d x \quad \forall z=(u, v) \in E$.

We know that $I_{\lambda, \mu}$ is not bounded below on $E$. From the following lemma, we have that $I_{\lambda, \mu}$ is bounded from below on the Nehari manifold $\mathcal{N}_{\lambda, \mu}$ defined in (9).

Lemma 3. The energy functional $I_{\lambda, \mu}$ is coercive and bounded below on $\mathcal{N}_{\lambda, \mu}$.

Proof. If $z=(u, v) \in \mathcal{N}_{\lambda, \mu}$, then by (10), the Hölder inequality, and the Sobolev embedding theorem, we get

$$
\begin{aligned}
I_{\lambda, \mu}(z)= & \frac{2^{*}-2}{2^{*} 2}\|z\|_{E}^{2}-\frac{2^{*}-q}{2^{*} q} K_{\lambda, \mu}(z) \\
\geq & \frac{1}{N}\|z\|_{E}^{2}-\frac{2^{*}-q}{2^{*} q} \gamma_{\infty} S^{-q / 2}|\Omega|^{\left(2^{*}-q\right) / 2^{*}} \\
& \times\left(\lambda^{2 /(2-q)}+\mu^{2 /(2-q)}\right)^{(2-q) / 2}\|z\|_{E}^{q} .
\end{aligned}
$$

Hence, we have that $I_{\lambda, \mu}$ is coercive and bounded below on $\mathcal{N}_{\lambda, \mu}$.

Define

$$
\Phi_{\lambda, \mu}(z)=\left\langle I_{\lambda, \mu}^{\prime}(z), z\right\rangle .
$$

Then, for $z \in \mathcal{N}_{\lambda, \mu}$,

$$
\begin{aligned}
& \left\langle\Phi_{\lambda, \mu}^{\prime}(z), z\right\rangle \\
& \quad=2\|z\|_{E}^{2}-q K_{\lambda, \mu}(z)-2^{*} \int_{\Omega} h(x)|u|^{\alpha} v^{\beta} d x \\
& =(2-q)\|z\|_{E}^{2}-\left(2^{*}-q\right) \int_{\Omega} h(x)|u|^{\alpha} v^{\beta} d x \\
& =\left(2^{*}-q\right) K_{\lambda, \mu}(z)-\left(2^{*}-2\right)\|z\|_{E}^{2} .
\end{aligned}
$$

We apply the method in [12]; let

$$
\begin{aligned}
& \mathcal{N}_{\lambda, \mu}^{+}=\left\{z \in \mathcal{N}_{\lambda, \mu} \mid\left\langle\Phi_{\lambda, \mu}^{\prime}(z), z\right\rangle>0\right\}, \\
& \mathcal{N}_{\lambda, \mu}^{0}=\left\{z \in \mathcal{N}_{\lambda, \mu} \mid\left\langle\Phi_{\lambda, \mu}^{\prime}(z), z\right\rangle=0\right\}, \\
& \mathcal{N}_{\lambda, \mu}^{-}=\left\{z \in \mathcal{N}_{\lambda, \mu} \mid\left\langle\Phi_{\lambda, \mu}^{\prime}(z), z\right\rangle<0\right\} .
\end{aligned}
$$

By using equality (17), we get that $K_{\lambda, \mu}(z)>0$ for $z \in$ $\mathcal{N}_{\lambda, \mu}^{+}$. Moreover, we have the following results.

Lemma 4. Let $\Lambda_{1}$ be a constant defined as in (8). If $0<$ $\lambda^{2 /(2-q)}+\mu^{2 /(2-q)}<\Lambda_{1}$, then $\mathcal{N}_{\lambda, \mu}^{0}=\emptyset$.

Proof. Assuming the contrary, there exist $\lambda, \mu>0$ with $0<$ $\lambda^{2 /(2-q)}+\mu^{2 /(2-q)}<\Lambda_{1}$ such that $\mathcal{N}_{\lambda, \mu}^{0} \neq \emptyset$. Then, by (16) and (17), for $u \in \mathscr{N}_{\lambda, \mu}^{0}$, we have

$$
\|z\|_{E}^{2}=\frac{2^{*}-q}{2-q} \int_{\Omega} h(x)|u|^{\alpha}|v|^{\beta} d x=\frac{2^{*}-q}{2^{*}-2} K_{\lambda, \mu}(z) .
$$

Using $(H 1)$ and both the Hölder and the Sobolev inequalities, we get

$$
\begin{gathered}
\|z\|_{E} \geq\left(\frac{2-q}{2^{*}-q} S^{2^{*} / 2}\right)^{1 /\left(2^{*}-2\right)}, \\
\|z\|_{E} \leq\left(\frac{2^{*}-q}{2^{*}-2} S^{-q / 2}|\Omega|^{\left(2^{*}-q\right) / 2^{*}} \gamma_{\infty}\right)^{1 /(2-q)} \\
\times\left(\lambda^{2 /(2-q)}+\mu^{2 /(2-q)}\right)^{1 / 2} .
\end{gathered}
$$

This implies

$$
\begin{aligned}
\lambda^{2 /(2-q)} & +\mu^{2 /(2-q)} \\
\geq & \left(\frac{2-q}{2^{*}-q}\right)^{2 /\left(2^{*}-2\right)}\left(\frac{\left(2^{*}-q\right) \gamma_{\infty}}{2^{*}-2}|\Omega|^{\left(2^{*}-q\right) / 2^{*}}\right)^{-2 /(2-q)} \\
& \times S^{N / 2+q /(2-q)}=\Lambda_{1},
\end{aligned}
$$

which is a contradiction.

For each $z \in E$ with $\int_{\Omega} h(x)|u|^{\alpha}|v|^{\beta} d x>0$, we write

$$
t_{\max }=\left(\frac{(2-q)\|z\|_{E}^{2}}{\left(2^{*}-q\right) \int_{\Omega} h(x)|u|^{\alpha}|v|^{\beta} d x}\right)^{1 /\left(2^{*}-2\right)}>0 .
$$

Then, the following lemma holds.

Lemma 5. Suppose that $0<\lambda^{2 /(2-q)}+\mu^{2 /(2-q)}<\Lambda_{1}$, and $z \in E$ with $\int_{\Omega} h(x)|u|^{\alpha}|v|^{\beta} d x>0$. Then, there exist unique $0<t^{+}<t_{\max }<t^{-}$such that $t^{+} z \in \mathcal{N}_{\lambda, \mu}^{+}, t^{-} z \in \mathcal{N}_{\lambda, \mu}^{-}$and

$$
\begin{gathered}
I_{\lambda, \mu}\left(t^{+} z\right)=\inf _{0 \leq t \leq t_{\max }} I_{\lambda, \mu}(t z) ; \\
I_{\lambda, \mu}\left(t^{-} z\right)=\sup _{t \geq 0} I_{\lambda, \mu}(t z) .
\end{gathered}
$$

Proof. This is similar to the proof of Hsu [14, Lemma 2.7].

Applying Lemma $4\left(\mathcal{N}_{\lambda, \mu}^{0}=\emptyset\right.$ for $0<\lambda^{2 /(2-q)}+\mu^{2 /(2-q)}<$ $\Lambda_{1}$ ), we write $\mathcal{N}_{\lambda, \mu}=\mathcal{N}_{\lambda, \mu}^{+} \cup \mathcal{N}_{\lambda, \mu}^{-}$and define

$$
\begin{aligned}
& \theta_{\lambda, \mu}=\inf _{z \in \mathcal{N}_{\lambda, \mu}} I_{\lambda, \mu}(z) ; \\
& \theta_{\lambda, \mu}^{+}=\inf _{z \in \mathcal{N}_{\lambda, \mu}^{+}} I_{\lambda, \mu}(z) ; \\
& \theta_{\lambda, \mu}^{-}=\inf _{z \in \mathcal{N}_{\lambda, \mu}^{-}} I_{\lambda, \mu}(z) .
\end{aligned}
$$

The following lemma shows that the minimizers on $\mathcal{N}_{\lambda, \mu}$ are usual critical points for $I_{\lambda, \mu}$.

Lemma 6. For the case when $\lambda \in\left(0, \Lambda_{1}\right)$, if $z_{0}$ is a local minimizer for $I_{\lambda, \mu}$ on $\mathcal{N}_{\lambda, \mu}$, then $I_{\lambda, \mu}^{\prime}\left(z_{0}\right)=0$ in $E^{-1}$. 
Proof. See Brown and Zhang [15, theorem 2.3].

Lemma 7. (i) If $0<\lambda^{2 /(2-q)}+\mu^{2 /(2-q)}<\Lambda_{1}$ and $z=(u, v) \epsilon$ $\mathcal{N}_{\lambda, \mu}^{+}$, then one has

$$
K_{\lambda, \mu}(z)>0, \quad I_{\lambda, \mu}(z)<0 .
$$

In particular, $\theta_{\lambda, \mu} \leq \theta_{\lambda, \mu}^{+}<0$.

(ii) If $0<\lambda^{2 /(2-q)}+\mu^{2 /(2-q)}<(q / 2)^{2 /(2-q)} \Lambda_{1}$ and $z=$ $(u, v) \in \mathcal{N}_{\lambda, \mu}^{-}$, then one has $u \neq \equiv, v \neq \equiv$ in $\Omega$,

$$
\|z\|_{E}>\left(\frac{2-q}{2^{*}-q}\right)^{1 /\left(2^{*}-2\right)} S^{N / 4}
$$

and $\theta_{\lambda, \mu}^{-}>d_{0}$ for some positive constant $d_{0}=d_{0}(\lambda, \mu, q, N, S$, $\left.\gamma_{\infty},|\Omega|\right)$.

Proof. (i) Let $z=(u, v) \in \mathcal{N}_{\lambda, \mu}^{+}$. By (16) and (17), we have

$$
\begin{gathered}
K_{\lambda, \mu}(z)>\frac{2^{*}-2}{2^{*}-q}\|z\|_{E}^{2}>0, \\
\frac{2-q}{2^{*}-q}\|z\|_{E}^{2}>\int_{\Omega} h(x)|u|^{\alpha}|v|^{\beta} d x .
\end{gathered}
$$

Then,

$$
\begin{aligned}
I_{\lambda, \mu}(z) & =\left(\frac{1}{2}-\frac{1}{q}\right)\|z\|_{E}^{2}+\left(\frac{1}{q}-\frac{1}{2^{*}}\right) \int_{\Omega} h(x)|u|^{\alpha}|v|^{\beta} d x \\
& <\left[\left(\frac{1}{2}-\frac{1}{q}\right)+\left(\frac{1}{q}-\frac{1}{2^{*}}\right) \frac{2-q}{2^{*}-q}\right]\|z\|_{E}^{2} \\
& =-\frac{2-q}{q N}\|z\|_{E}^{2}<0 .
\end{aligned}
$$

By the definition of $\theta_{\lambda, \mu}, \theta_{\lambda, \mu}^{+}$, we deduce that $\theta_{\lambda, \mu} \leq \theta_{\lambda, \mu}^{+}<0$.

(ii) Let $z \in \mathcal{N}_{\lambda, \mu}^{-}$; by (16) and the Hölder and the Sobolev inequalities, we get

$$
\frac{2-q}{2^{*}-q}\|z\|_{E}^{2}<\int_{\Omega} h(x)|u|^{\alpha}|v|^{\beta} d x \leq S^{-2^{*} / 2}\|z\|_{E}^{2^{*}} .
$$

This implies

$$
\begin{aligned}
\int_{\Omega} h(x)|u|^{\alpha}|v|^{\beta} d x & >\frac{2^{*}-q}{2-q}\|z\|_{E} \\
& >\left(\frac{2-q}{2^{*}-q}\right)^{\frac{1}{2^{*}-2}} S \frac{N}{4} \quad \forall z \in \mathcal{N}_{\lambda, \mu}^{-} .
\end{aligned}
$$

By (13) and (31), we obtain that $u \neq \equiv 0, v \neq \equiv$ in $\Omega$, and

$$
\begin{aligned}
& I_{\lambda, \mu}(z) \\
& \geq\|z\|_{E}^{q}\left[\frac{1}{N}\|z\|_{E}^{2-q}-\left(\frac{2^{*}-q}{2^{*} q}\right) \gamma_{\infty} S^{-q / 2}|\Omega|^{\left(2^{*}-q\right) / 2^{*}}\right. \\
& \left.\quad \times\left(\lambda^{2 /(2-q)}+\mu^{2 /(2-q)}\right)^{(2-q) / 2}\right] \\
& >\left(\frac{2-q}{2^{*}-q}\right)^{q /\left(2^{*}-2\right)} S^{q N / 4} S^{(2-q) N / 4} \\
& \times\left[\frac{1}{N}\left(\frac{2-q}{2^{*}-q}\right)^{(2-q) /\left(2^{*}-2\right)}\right. \\
& \quad-\left(\frac{2^{*}-q}{2^{*} q}\right) \gamma_{\infty} S^{-q / 2}|\Omega|^{\left(2^{*}-q\right) / 2^{*}} \\
& \left.\times\left(\lambda^{2 /(2-q)}+\mu^{2 /(2-q)}\right)^{(2-q) / 2}\right] .
\end{aligned}
$$

Thus, if $0<\lambda^{2 /(2-q)}+\mu^{2 /(2-q)}<(q / 2)^{2 /(2-q)} \Lambda_{1}$, for all $z \in$ $\mathcal{N}_{\lambda, \mu}^{-}$, then

$$
I_{\lambda, \mu}(z) \geq d_{0}\left(\lambda, \mu, q, N, S, \gamma_{\infty},|\Omega|\right)>0
$$

\section{Existence of a Ground State Solution}

First of all, we define the Palais-Smale (denote by (PS)) sequences and (PS)-condition in $E$ for $I_{\lambda, \mu}$ as follows.

Definition 8. (i) For $\gamma \in \mathbb{R}$, a sequence $\left\{z_{n}\right\}$ is a (PS) $\gamma^{-}$ sequence in $E$ for $I_{\lambda, \mu}$ if $I_{\lambda, \mu}\left(z_{n}\right)=\gamma+o_{n}(1)$ and $I_{\lambda, \mu}^{\prime}\left(z_{n}\right)=$ $o_{n}(1)$ strongly in $E^{-1}$ as $n \rightarrow \infty$.

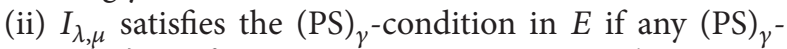
sequence $\left\{z_{n}\right\}$ in $E$ for $I_{\lambda, \mu}$ contains a convergent subsequence.

Proof of Theorem 1. Using the same argument as in $\mathrm{Wu}$ [16, Proposition 9] or Hsu [14, Proposition 3.3], there exists a minimizing sequence $\left\{z_{n}\right\}$ for $I_{\lambda, \mu}$ on $\mathcal{N}_{\lambda, \mu}$ such that

$$
I_{\lambda, \mu}\left(z_{n}\right)=\theta_{\lambda, \mu}+o_{n}(1), \quad I_{\lambda, \mu}^{\prime}\left(z_{n}\right)=o_{n}(1) \text { in } E^{-1} .
$$

Since $I_{\lambda, \mu}$ is coercive on $\mathcal{N}_{\lambda, \mu}$ (see Lemma 3), we get that $\left\{z_{n}\right\}$ is bounded in $E$. Then, there exist a subsequence $\left\{z_{n}=\right.$ $\left.\left(u_{n}, v_{n}\right)\right\}$ and $z_{\lambda, \mu}^{1}=\left(u_{\lambda, \mu}^{1}, v_{\lambda, \mu}^{1}\right) \in E$ such that

$$
u_{n} \rightarrow u_{\lambda, \mu}^{1}, \quad v_{n} \rightarrow v_{\lambda, \mu}^{1} \quad \text { weakly in } H_{0}^{1}(\Omega),
$$

$u_{n} \longrightarrow u_{\lambda, \mu}^{1}, \quad v_{n} \longrightarrow v_{\lambda, \mu}^{1} \quad$ almost everywhere in $\Omega$,

$u_{n} \longrightarrow u_{\lambda, \mu}^{1}, \quad v_{n} \longrightarrow v_{\lambda, \mu}^{1} \quad$ strongly in $L^{s}(\Omega) \forall 1 \leq s<2^{*}$. 
This implies

$$
K_{\lambda, \mu}\left(z_{n}\right)=K_{\lambda, \mu}\left(z_{\lambda, \mu}^{1}\right)+o_{n}(1) \quad \text { as } n \longrightarrow \infty .
$$

First, we claim that $z_{\lambda, \mu}^{1}$ is a nontrivial solution of $\left(P_{\lambda, \mu}\right)$. By (34) and (35), it is easy to verify that $z_{\lambda, \mu}^{1}$ is a weak solution of $\left(P_{\lambda, \mu}\right)$. From $z_{n} \in \mathcal{N}_{\lambda, \mu}$ and (12), we deduce that

$$
K_{\lambda, \mu}\left(z_{n}\right)=\frac{q\left(2^{*}-2\right)}{2\left(2^{*}-q\right)}\left\|z_{n}\right\|_{E}^{2}-\frac{2^{*} q}{2^{*}-q} I_{\lambda, \mu}\left(z_{n}\right) .
$$

Let $n \rightarrow \infty$ in (37); by (34), (36), and $\theta_{\lambda, \mu}<0$, we get

$$
K_{\lambda, \mu}\left(z_{\lambda, \mu}^{1}\right) \geq-\frac{2^{*} q}{2^{*}-q} \theta_{\lambda, \mu}>0 .
$$

Thus, $z_{\lambda, \mu}^{1} \in \mathcal{N}_{\lambda, \mu}$ is a nontrivial solution of $\left(P_{\lambda, \mu}\right)$. Now, we prove that $z_{n} \rightarrow z_{\lambda, \mu}^{1}$ strongly in $E$ and $I_{\lambda, \mu}\left(z_{\lambda, \mu}^{1}\right)=\theta_{\lambda, \mu}$. By (37), if $z \in \mathcal{N}_{\lambda, \mu}$, then

$$
I_{\lambda, \mu}(z)=\frac{1}{N}\|z\|_{E}^{2}-\frac{2^{*}-q}{2^{*} q} K_{\lambda, \mu}(z) .
$$

In order to prove that $I_{\lambda, \mu}\left(z_{\lambda, \mu}^{1}\right)=\theta_{\lambda, \mu}$, it suffices to recall that $z_{\lambda, \mu}^{1} \in \mathcal{N}_{\lambda, \mu}$, by (39) and applying Fatou's lemma to get

$$
\begin{aligned}
\theta_{\lambda, \mu} & \leq I_{\lambda, \mu}\left(z_{\lambda, \mu}^{1}\right)=\frac{1}{N}\left\|z_{\lambda, \mu}^{1}\right\|_{E}^{2}-\frac{2^{*}-q}{2^{*} q} K_{\lambda, \mu}\left(z_{\lambda, \mu}^{1}\right) \\
& \leq \liminf _{n \rightarrow \infty}\left(\frac{1}{N}\left\|z_{n}\right\|_{E}^{2}-\frac{2^{*}-q}{2^{*} q} K_{\lambda, \mu}\left(z_{n}\right)\right) \\
& \leq \liminf _{n \rightarrow \infty} I_{\lambda, \mu}\left(z_{n}\right)=\theta_{\lambda, \mu} .
\end{aligned}
$$

This implies that $I_{\lambda, \mu}\left(z_{\lambda, \mu}^{1}\right)=\theta_{\lambda, \mu}$ and $\lim _{n \rightarrow \infty}\left\|z_{n}\right\|_{E}^{2}=$ $\left\|z_{\lambda, \mu}^{1}\right\|_{E}^{2}$. Let $\widetilde{z}_{n}=z_{n}-z_{\lambda, \mu}^{1}$; then Brézis-Lieb lemma [17] implies

$$
\left\|\widetilde{z}_{n}\right\|_{E}^{2}=\left\|z_{n}\right\|_{E}^{2}-\left\|z_{\lambda, \mu}^{1}\right\|_{E}^{2}
$$

Therefore, $z_{n} \rightarrow z_{\lambda, \mu}^{1}$ strongly in E. Since $I_{\lambda, \mu}\left(z_{\lambda, \mu}^{1}\right)=$ $I_{\lambda, \mu}\left(\left|z_{\lambda, \mu}^{1}\right|\right)=\theta_{\lambda, \mu}$ and $\left|z_{\lambda, \mu}^{1}\right| \in \mathcal{N}_{\lambda, \mu}^{+}$, by Lemma 6 we may assume that $z_{\lambda, \mu}^{1}$ is a nontrivial nonnegative solution of $\left(P_{\lambda, \mu}\right)$. By an argument of Hsu [18, Lemma 4.2], we can deduce that $u_{\lambda, \mu}^{1} \neq \equiv 0$ and $v_{\lambda, \mu}^{1} \not \equiv 0$ in $\Omega$. Finally, from the maximum principle [19], we deduce that $z_{\lambda, \mu}^{1}$ is positive in $\Omega$.

Remark 9. $z_{\lambda, \mu}^{1} \in \mathcal{N}_{\lambda, \mu}^{+}$and $I_{\lambda, \mu}\left(z_{\lambda, \mu}^{1}\right)=\theta_{\lambda, \mu}=\theta_{\lambda, \mu}^{+}$.

Proof. We claim that $z_{\lambda, \mu}^{1} \in \mathcal{N}_{\lambda, \mu}^{+}$. On the contrary, assume that $z_{\lambda, \mu}^{1} \in \mathscr{N}_{\lambda, \mu}^{-}\left(\mathscr{N}_{\lambda, \mu}^{0}=\emptyset\right.$ for $\left.\lambda^{2 /(2-q)}+\mu^{2 /(2-q)} \in\left(0, \Lambda_{1}\right)\right)$; then by Lemma 5 , there exist unique $t_{1}^{+}$and $t_{1}^{-}$such that $t_{1}^{+} z_{\lambda, \mu}^{1} \in \mathcal{N}_{\lambda, \mu}^{+}$and $t_{1}^{-} z_{\lambda, \mu}^{1} \in \mathcal{N}_{\lambda, \mu}^{-}$. In particular, we have $t_{1}^{+}<t_{1}^{-}=1$. Since

$$
\frac{d}{d t} I_{\lambda, \mu}\left(t_{1}^{+} z_{\lambda, \mu}^{1}\right)=0, \quad \frac{d^{2}}{d t^{2}} I_{\lambda, \mu}\left(t_{1}^{+} z_{\lambda, \mu}^{1}\right)>0,
$$

there exists $t_{1}^{+}<\bar{t} \leq t_{1}^{-}$such that $I_{\lambda, \mu}\left(t_{1}^{+} z_{\lambda, \mu}^{1}\right)<I_{\lambda, \mu}\left(\bar{t} z_{\lambda, \mu}^{1}\right)$. By Lemma 5,

$$
I_{\lambda, \mu}\left(t_{1}^{+} z_{\lambda, \mu}^{1}\right)<I_{\lambda, \mu}\left(\bar{t} z_{\lambda, \mu}^{1}\right) \leq I_{\lambda, \mu}\left(t_{1}^{-} z_{\lambda, \mu}^{1}\right)=I_{\lambda, \mu}\left(z_{\lambda, \mu}^{1}\right),
$$

which is a contradiction. Hence, $z_{\lambda, \mu}^{1} \in \mathcal{N}_{\lambda, \mu}^{+}$and $I_{\lambda, \mu}\left(z_{\lambda, \mu}^{1}\right)=$ $\theta_{\lambda, \mu}=\theta_{\lambda, \mu}^{+}$

\section{Existence of $k+1$ Solutions}

Throughout this section, $(H 1)-(H 2)$ will be assumed. First of all, we want to show that $I_{\lambda, \mu}$ satisfies the (PS) $\gamma_{\gamma}$-condition in $E$ for $\gamma \in\left(-\infty,(1 / N)\left(S_{\alpha, \beta}\right)^{N / 2}-C_{0}\left(\lambda^{2 /(2-q)}+\mu^{2 /(2-q)}\right)\right)$, where $C_{0}$ is defined in the following lemma.

Lemma 10. If $\left\{z_{n}\right\} \subset E$ is a $(P S)_{\gamma}$-sequence for $I_{\lambda, \mu}$ with $z_{n} \rightarrow$ $z$ weakly in $E$, then $I_{\lambda, \mu}^{\prime}(z)=0$ and there exists a constant $C_{0}=$ $C_{0}\left(q, N, S, \gamma_{\infty},|\Omega|\right)>0$ such that $I_{\lambda, \mu}(z) \geq-C_{0}\left(\lambda^{2 /(2-q)}+\right.$ $\left.\mu^{2 /(2-q)}\right)$.

Proof. Let $z_{n}=\left(u_{n}, v_{n}\right)$ and $z=(u, v)$. If $\left\{z_{n}\right\}$ is a (PS) $\gamma^{-}$ sequence for $I_{\lambda, \mu}$ with $z_{n} \rightarrow z$ weakly in $E$, it is easy to check that $I_{\lambda, \mu}^{\prime}(z)=0$ in $E^{-1}$. Then, we get $\left\langle I_{\lambda, \mu}^{\prime}(z), z\right\rangle=0$; that is, $\int_{\Omega} h(x)|u|^{\alpha}|v|^{\beta} d x=\|z\|_{E}^{2}-K_{\lambda, \mu}(z)$. Thus, by (13), the Hölder, the Young, and the Sobolev inequalities, we have

$$
\begin{aligned}
I_{\lambda, \mu}(z) \geq & \frac{1}{N}\|z\|_{E}^{2}-\frac{2^{*}-q}{2^{*} q} \gamma_{\infty} S^{-q / 2}|\Omega|^{\left(2^{*}-q\right) / 2^{*}} \\
& \times\left(\lambda^{2 /(2-q)}+\mu^{2 /(2-q)}\right)^{(2-q) / 2}\|z\|_{E}^{q} \\
\geq & \frac{1}{N}\|z\|_{E}^{2}-\frac{1}{N}\|z\|_{E}^{2}-C_{0}\left(\lambda^{2 /(2-q)}+\mu^{2 /(2-q)}\right) \\
= & -C_{0}\left(\lambda^{2 /(2-q)}+\mu^{2 /(2-q)}\right)
\end{aligned}
$$

where $C_{0}=C_{0}\left(q, N, S, \gamma_{\infty},|\Omega|\right)>0$.

Lemma 11. If $\left\{z_{n}\right\} \subset E$ is a $(P S)_{\gamma}$-sequence for $I_{\lambda, \mu}$, then $\left\{z_{n}\right\}$ is bounded in $E$.

Proof. See Hsu and Lin [8, Lemma 2.3].

Recall that

$$
S_{\alpha, \beta}=\inf _{u, v \in H_{0}^{1}(\Omega) \backslash\{0\}} \frac{\|(u, v)\|_{E}^{2}}{\left(\int_{\Omega}|u|^{\alpha}|v|^{\beta} d x\right)^{2 /(\alpha+\beta)}},
$$

and let

$$
c^{*}=\frac{1}{N}\left(S_{\alpha, \beta}\right)^{N / 2}-C_{0}\left(\lambda^{2 /(2-q)}+\mu^{2 /(2-q)}\right),
$$

where $C_{0}>0$ is given in Lemma 10 .

Lemma 12. $I_{\lambda, \mu}$ satisfies the $(P S)_{\gamma}$-condition in $E$ for $\gamma \in$ $\left(-\infty, c^{*}\right)$. 
Proof. Let $\left\{z_{n}\right\} \subset E$ be a (PS) $)_{\gamma}$-sequence for $I_{\lambda, \mu}$ with $\gamma \in$ $\left(-\infty, c^{*}\right)$. Write $z_{n}=\left(u_{n}, v_{n}\right)$. We know from Lemma 11 that $\left\{z_{n}\right\}$ is bounded in $E$, and then $z_{n} \rightarrow z=(u, v)$ weakly up to a subsequence; $z$ is a critical point of $I_{\lambda, \mu}$. Furthermore, we may assume that $u_{n} \rightarrow u, v_{n} \rightarrow v$ weakly in $H_{0}^{1}(\Omega)$ and $u_{n} \rightarrow u$, $v_{n} \rightarrow v$ strongly in $L^{s}(\Omega)$ for all $1 \leq s<2^{*}$, and $u_{n} \rightarrow u$, $v_{n} \rightarrow v$ a.e. on $\Omega$. Hence, we have that $I_{\lambda, \mu}^{\prime}(z)=0$ and

$$
K_{\lambda, \mu}\left(z_{n}\right)=K_{\lambda, \mu}(z)+o_{n}(1) .
$$

Let $\widetilde{u}_{n}=u_{n}-u, \widetilde{v}_{n}=v_{n}-v$ and $\widetilde{z}_{n}=\left(\widetilde{u}_{n}, \widetilde{v}_{n}\right)$. Then, we obtain

$$
\left\|\widetilde{z}_{n}\right\|_{E}^{2}=\left\|z_{n}\right\|_{E}^{2}-\|z\|_{E}^{2}+o_{n}(1),
$$

and by an argument of Han [20, Lemma 2.1],

$$
\begin{aligned}
& \int_{\Omega} h(x)\left|\widetilde{u}_{n}\right|^{\alpha}\left|\widetilde{v}_{n}\right|^{\beta} d x \\
& =\int_{\Omega} h(x)\left|u_{n}\right|^{\alpha}\left|v_{n}\right|^{\beta} d x \\
& \quad-\int_{\Omega} h(x)|u|^{\alpha}|v|^{\beta} d x+o_{n}(1) .
\end{aligned}
$$

Since $I_{\lambda, \mu}\left(z_{n}\right)=\gamma+o_{n}(1), I_{\lambda, \mu}^{\prime}\left(z_{n}\right)=o_{n}(1)$ in $E^{-1}$ and (47)(49), we deduce that

$$
\begin{gathered}
\frac{1}{2}\left\|\widetilde{z}_{n}\right\|_{E}^{2}-\frac{1}{2^{*}} \int_{\Omega} h(x)\left|\widetilde{u}_{n}\right|^{\alpha}\left|\widetilde{v}_{n}\right|^{\beta} d x=\gamma-I_{\lambda, \mu}(z)+o_{n}(1) \\
\left\|\widetilde{z}_{n}\right\|_{E}^{2}-\int_{\Omega} h(x)\left|\widetilde{u}_{n}\right|^{\alpha}\left|\widetilde{v}_{n}\right|^{\beta} d x=o_{n}(1)
\end{gathered}
$$

Hence, we may assume that

$$
\left\|\widetilde{z}_{n}\right\|_{E}^{2} \longrightarrow l, \quad \int_{\Omega} h(x)\left|\widetilde{u}_{n}\right|^{\alpha}\left|\widetilde{v}_{n}\right|^{\beta} d x \longrightarrow l .
$$

Assume that $l \neq 0$; by the definition of $S_{\alpha, \beta},|h|_{L^{\infty}(\Omega)}=1$ and (52), we obtain

$$
\begin{aligned}
\left.S_{\alpha, \beta}\right|^{2 / 2^{*}} & =S_{\alpha, \beta_{n}} \lim _{n \rightarrow \infty}\left(\int_{\Omega} h(x)\left|\tilde{u}_{n}\right|^{\alpha}\left|\tilde{v}_{n}\right|^{\beta} d x\right)^{2 / 2^{*}} \\
& \leq|h|_{L^{\infty}(\Omega)}^{2 / 2^{*}} \lim _{n \rightarrow \infty}\left\|\tilde{z}_{n}\right\|^{2}=l,
\end{aligned}
$$

which implies that $l \geq\left(S_{\alpha, \beta}\right)^{N / 2}$. In addition, from Lemma 10 , (50), and (52), we get

$$
\begin{aligned}
\gamma & =\left(\frac{1}{2}-\frac{1}{2^{*}}\right) l+I_{\lambda, \mu}(z) \\
& \geq \frac{1}{N}\left(S_{\alpha, \beta}\right)^{N / 2}-C_{0}\left(\lambda^{2 /(2-q)}+\mu^{2 /(2-q)}\right),
\end{aligned}
$$

which is a contradiction. Hence, $l=0$; that is, $z_{n} \rightarrow z$ strongly in $E$. that

From assumption $(H 2)$, we can choose $r_{0} \in(0,1)$ such

$$
\overline{B_{r_{0}}\left(a_{i}\right)} \cap \overline{B_{r_{0}}\left(a_{j}\right)}=\emptyset \quad \text { for } i \neq j, 1 \leq i, j \leq k,
$$

and $\cup_{i=1}^{k} \overline{B_{r_{0}}\left(a_{i}\right)} \subset \Omega$, where $\overline{B_{r_{0}}\left(a_{i}\right)}=\left\{x \in \mathbb{R}^{N}|| x-a_{i} \mid \leq r_{0}\right\}$ and $h\left(a_{i}\right)=|h|_{\infty}=1$ for $1 \leq i \leq k$.

Define

$Q_{i}(z)=\frac{\int_{\Omega} \psi_{i}(x)\left(|\nabla u|^{2}+|\nabla v|^{2}\right) d x}{\int_{\Omega}\left(|\nabla u|^{2}+|\nabla v|^{2}\right) d x}, \quad z=(u, v) \in E \backslash\{0\}$,

where $\psi_{i}(x)=\min \left\{1,\left|x-a_{i}\right|\right\}, i=1,2 \ldots, k$.

Then, we have the following separation result.

Lemma 13. If $Q_{i}(z) \leq r_{0} / 3$ and $Q_{j}(z) \leq r_{0} / 3$ for $z \in E \backslash\{0\}$, then $i=j$.

Proof. For any $z \in E \backslash\{0\}$ satisfying $Q_{i}(z) \leq r_{0} / 3(1 \leq i \leq k)$, we get

$$
\begin{aligned}
\frac{r_{0}}{3}\|z\|_{E}^{2} & \geq \int_{\Omega} \psi_{i}(x)\left(|\nabla u|^{2}+|\nabla v|^{2}\right) d x \\
& \geq \int_{\Omega \backslash B_{r_{0}}\left(a_{i}\right)} \psi_{i}(x)\left(|\nabla u|^{2}+|\nabla v|^{2}\right) d x \\
& \geq r_{0} \int_{\Omega \backslash B_{r_{0}}\left(a_{i}\right)}\left(|\nabla u|^{2}+|\nabla v|^{2}\right) d x,
\end{aligned}
$$

which implies that

$$
\|z\|_{E}^{2} \geq 3 \int_{\Omega \backslash B_{r_{0}}\left(a_{i}\right)}\left(|\nabla u|^{2}+|\nabla v|^{2}\right) d x, \quad 1 \leq i \leq k .
$$

Hence, from (58), we obtain

$$
\begin{aligned}
2\|z\|_{E}^{2} \geq 3\left(\int_{\Omega \backslash B_{r_{0}}\left(a_{i}\right)}\left(|\nabla u|^{2}+|\nabla v|^{2}\right) d x\right. \\
\left.\quad+\int_{\Omega \backslash B_{r_{0}}\left(a_{j}\right)}\left(|\nabla u|^{2}+|\nabla v|^{2}\right) d x\right) \\
\geq 3\|z\|_{E}^{2} \quad \text { if } i \neq j,
\end{aligned}
$$

which is a contradiction.

For $i=1,2, \ldots, k$, we set

$$
\begin{aligned}
\mathcal{N}_{\lambda, \mu}^{i} & =\left\{u \in \mathcal{N}_{\lambda, \mu}^{-} \mid Q_{i}(z)<\frac{r_{0}}{3}\right\}, \\
\partial \mathcal{N}_{\lambda, \mu}^{i} & =\left\{u \in \mathcal{N}_{\lambda, \mu}^{-} \mid Q_{i}(z)=\frac{r_{0}}{3}\right\},
\end{aligned}
$$

and define

$$
\theta_{\lambda, \mu}^{i}=\inf _{\mathcal{N}_{\lambda, \mu}^{i}} I_{\lambda, \mu}(z), \quad \widetilde{\theta}_{\lambda, \mu}^{i}=\inf _{\partial \mathcal{N}_{\lambda, \mu}^{i}} I_{\lambda, \mu}(z)
$$

Recall that the best Sobolev constant $S$ is defined as

$$
S=\inf _{u \in D^{1,2}\left(\mathbb{R}^{N}\right) \backslash\{0\}} \frac{\int_{\mathbb{R}^{N}}|\nabla u|^{2} d x}{\left(\int_{\mathbb{R}^{N}}|u|^{2^{*}} d x\right)^{2 / 2^{*}}} .
$$


It is well known that

$$
U(x)=\frac{[N(N-2)]^{(N-2) / 4}}{\left[1+|x|^{2}\right]^{(N-2) / 2}}
$$

is a minimizer of $S$, and $|\nabla U|_{L^{2}\left(\mathbb{R}^{N}\right)}^{2}=|U|_{L^{2^{*}\left(\mathbb{R}^{N}\right)}}^{2^{*}}=S^{N / 2}$. Fix a maximum point $a_{i}$ of $h(1 \leq i \leq k)$. Let $\eta_{i} \in C_{0}^{\infty}(\Omega)$ be a cut-off function such that $0 \leq \eta_{i} \leq 1,\left|\nabla \eta_{i}\right| \leq C$, and $\eta_{i}(x)=1$ for $\left|x-a_{i}\right|<r_{0} / 2, \eta_{i}(x)=0$ for $\left|x-a_{i}\right|>r_{0}$. We define

$$
u_{\varepsilon}^{i}(x)=\varepsilon^{(2-N) / 2} \eta_{i}(x) U\left(\frac{x-a_{i}}{\varepsilon}\right)=\frac{c_{1} \varepsilon^{(N-2) / 2} \eta_{i}(x)}{\left[\varepsilon^{2}+\left|x-a_{i}\right|^{2}\right]^{(N-2) / 2}},
$$

where $c_{1}=[N(N-2)]^{(N-2) / 4}$ and $\varepsilon>0$.

From now on, we assume that $N /(N-2)<q<2$ and $N>4$.

Lemma 14. There exist $\varepsilon_{0}>0, \Lambda_{2} \in\left(0,(q / 2)^{2 /(2-q)} \Lambda_{1}\right)$, such that for $\varepsilon \in\left(0, \varepsilon_{0}\right)$ and $\lambda^{2 /(2-q)}+\mu^{2 /(2-q)} \in\left(0, \Lambda_{2}\right)$, one has

$$
\sup _{t \geq 0} I_{\lambda, \mu}\left(t \sqrt{\alpha} u_{\varepsilon}^{i}, t \sqrt{\beta} u_{\varepsilon}^{i}\right)<c^{*} \quad \text { uniformly in } i,
$$

where $c^{*}$ is the positive constant given in Lemma 12.

In particular, $0<\theta_{\lambda, \mu}^{-} \leq \theta_{\lambda, \mu}^{i}<c^{*}$ for all $1 \leq i \leq k$.

Proof. It is well known that (or see Brézis and Nirenberg [21], Cheng and $\mathrm{Ma}$ [22, Lemma 3.2], Struwe [23], and Willem [24, Lemma 1.46]) as $\varepsilon \rightarrow 0^{+}$,

$$
\begin{aligned}
& \left|u_{\varepsilon}^{i}\right|_{L^{2^{*}(\Omega)}}^{2}=|U|_{L^{2^{*}}\left(\mathbb{R}^{N}\right)}^{2}+O\left(\varepsilon^{N-2}\right), \\
& \left|\nabla u_{\varepsilon}^{i}\right|_{L^{2}(\Omega)}^{2}|\nabla U|_{L^{2}\left(\mathbb{R}^{N}\right)}^{2}+O\left(\varepsilon^{N-2}\right) .
\end{aligned}
$$

For $N /(N-2)<q<2, N>4$ and $\varepsilon<r_{0} / 2$,

$$
\begin{aligned}
\left|u_{\varepsilon}^{i}\right|_{L^{q}(\Omega)}^{q} & =\int_{B_{r_{0} / 2}\left(a_{i}\right)}\left[\varepsilon^{(2-N) / 2} U\left(\frac{x-a_{i}}{\varepsilon}\right)\right]^{q} d x+O\left(\varepsilon^{N-2}\right) \\
& \geq C \varepsilon^{\theta}+O\left(\varepsilon^{N-2}\right), \quad \text { where } \theta=N-\frac{(N-2) q}{2} .
\end{aligned}
$$

Set $\bar{z}_{\varepsilon}^{i}=\left(\sqrt{\alpha} u_{\varepsilon}^{i}, \sqrt{\beta} u_{\varepsilon}^{i}\right)$. By Lemma 5 , there exists $t_{\varepsilon}^{i}>0$ such that $z_{\varepsilon}^{i}=t_{\varepsilon}^{i} \bar{z}_{\varepsilon}^{i} \in \mathcal{N}_{\lambda, \mu}^{-}$for $0<\lambda^{2 /(2-q)}+\mu^{2 /(2-q)}<\Lambda_{1}$. Furthermore,

$$
\begin{aligned}
Q_{i}\left(z_{\varepsilon}^{i}\right) & =\frac{\int_{\Omega} \psi_{i}(x)\left|\nabla u_{\varepsilon}^{i}\right|^{2} d x}{\int_{\Omega}\left|\nabla u_{\varepsilon}^{i}\right|^{2} d x} \\
& =\frac{\int_{\left(\Omega-a_{i}\right) / \varepsilon} \psi_{i}\left(a_{i}+\varepsilon y\right)\left|\nabla\left(\eta_{i}\left(a_{i}+\varepsilon y\right) U(y)\right)\right|^{2} d y}{\int_{\left(\Omega-a_{i}\right) / \varepsilon}\left|\nabla\left(\eta_{i}\left(a_{i}+\varepsilon y\right) U(y)\right)\right|^{2} d y} \\
& \longrightarrow \psi_{i}\left(a_{i}\right)=0 \quad \text { as } \varepsilon \longrightarrow 0 .
\end{aligned}
$$

Hence, there exists $\bar{\varepsilon}_{0}>0$ for any

$$
\varepsilon \in\left(0, \bar{\varepsilon}_{0}\right), \quad Q_{i}\left(z_{\varepsilon}^{i}\right)<\frac{r_{0}}{3},
$$

which implies

$$
z_{\varepsilon}^{i}=t_{\varepsilon}^{i} \bar{z}_{\varepsilon}^{i} \in \mathscr{N}_{\lambda, \mu}^{i} \quad \text { for } \varepsilon \in\left(0, \bar{\varepsilon}_{0}\right),
$$

and then

$$
\theta_{\lambda, \mu}^{-} \leq \theta_{\lambda, \mu}^{i} \leq I_{\lambda, \mu}\left(z_{\varepsilon}^{i}\right) \leq \sup _{t \geq 0} I_{\lambda, \mu}\left(t t_{\varepsilon}^{i} \bar{z}_{\varepsilon}^{i}\right)=\sup _{t \geq 0} I_{\lambda, \mu}\left(t \bar{z}_{\varepsilon}^{i}\right) .
$$

First, we consider the functional $I_{0,0}: E \rightarrow \mathbb{R}$ defined by

$$
I_{0,0}(u, v)=\frac{1}{2}\|(u, v)\|_{E}^{2}-\frac{1}{2^{*}} \int_{\Omega} h(x)|u|^{\alpha}|v|^{\beta} d x .
$$

Step I. Show that $\sup _{t \geq 0} I_{0,0}\left(\bar{z}_{\varepsilon}^{i}\right) \leq(1 / N)\left(S_{\alpha, \beta}\right)^{N / 2}+O\left(\varepsilon^{N-2}\right)$. According to condition (H2), we conclude that

$$
\begin{aligned}
& \left.\left|\int_{\Omega} h(x)\right| u_{\varepsilon}^{i}(x)\right|^{2^{*}} d x-\int_{\Omega} h\left(a_{i}\right)\left|u_{\varepsilon}^{i}(x)\right|^{2^{*}} d x \mid \\
& \leq \int_{\Omega}\left|h(x)-h\left(a_{i}\right)\right|\left|u_{\varepsilon}^{i}(x)\right|^{2^{*}} d x \\
& \quad=O\left(\int_{B_{r_{0}}\left(a_{i}\right)}\left|x-a_{i}\right|^{\sigma}\left|u_{\varepsilon}^{i}(x)\right|^{2^{*}} d x\right) \\
& =O\left(\varepsilon^{\sigma}\right) .
\end{aligned}
$$

From (66), (74), $h\left(a_{i}\right)=1$, and $\sigma \geq N-2$, we can deduce that

$$
\begin{aligned}
\left(\int_{\Omega} h\right. & \left.h(x)\left|u_{\varepsilon}^{i}(x)\right|^{2^{*}} d x\right)^{2 / 2^{*}} \\
& =\left(\left|u_{\varepsilon}^{i}\right|_{L^{2^{*}}(\Omega)}^{2^{*}}+O\left(\varepsilon^{\sigma}\right)\right)^{2 / 2^{*}} \\
& =\left|u_{\varepsilon}^{i}\right|_{L^{2^{*}}(\Omega)}^{2}+O\left(\varepsilon^{\sigma}\right) \\
& =|U|_{L^{2^{*}}\left(\mathbb{R}^{N}\right)}^{2}+O\left(\varepsilon^{N-2}\right) .
\end{aligned}
$$

Using (67) and (75), then

$$
\begin{gathered}
\frac{\left|\nabla u_{\varepsilon}^{i}\right|_{L^{2}(\Omega)}^{2}}{\left(\int_{\Omega} h(x)\left|u_{\varepsilon}^{i}(x)\right|^{2^{*}} d x\right)^{2 / 2^{*}}} \\
=\frac{|\nabla U|_{L^{2}\left(\mathbb{R}^{N}\right)}^{2}+O\left(\varepsilon^{N-2}\right)}{|U|_{L^{2^{*}}\left(\mathbb{R}^{N}\right)}^{2}+O\left(\varepsilon^{N-2}\right)} \\
=S+O\left(\varepsilon^{N-2}\right) .
\end{gathered}
$$

Since

$$
\begin{aligned}
\sup _{t \geq 0}( & \left.\frac{A}{2} t^{2}-\frac{B}{2^{*}} t^{2^{*}}\right) \\
& =\frac{1}{N}\left(\frac{A}{B^{2 / 2^{*}}}\right)^{N / 2}, \text { for any } A>0, B>0,
\end{aligned}
$$


by (7) and (76), we conclude that

$$
\begin{aligned}
\sup _{t \geq 0} I_{0,0}\left(t \bar{z}_{\varepsilon}^{i}\right) & \\
& =\frac{1}{N}\left(\frac{(\alpha+\beta)\left|\nabla u_{\varepsilon}^{i}\right|_{L^{2}(\Omega)}^{2}}{\left(\alpha^{\alpha / 2} \beta^{\beta / 2} \int_{\Omega} h(x)\left|u_{\varepsilon}^{i}(x)\right|^{2^{*}} d x\right)^{2 / 2^{*}}}\right)^{N / 2} \\
& \leq \frac{1}{N}\left(S_{\alpha, \beta}\right)^{N / 2}+O\left(\varepsilon^{N-2}\right) .
\end{aligned}
$$

Step II. Let $C_{0}$ be the positive constant given in Lemma 10. We can choose $\delta_{1}>0$ such that for all $0<\lambda^{2 /(2-q)}+\mu^{2 /(2-q)}<\delta_{1}$, we have

$$
c^{*}=\frac{1}{N}\left(S_{\alpha, \beta}\right)^{N / 2}-C_{0}\left(\lambda^{2 /(2-q)}+\mu^{2 /(2-q)}\right)>0 .
$$

Since $I_{\lambda, \mu}$ is continuous in $E, I_{\lambda, \mu}(0)=0$, and $\left\{\bar{z}_{\varepsilon}^{i}\right\}$ is uniformly bounded in $E$ for any $0<\varepsilon<\min \left\{\bar{\varepsilon}_{0}, r_{0} / 2\right\}$ (see (67)), then there exists $t_{0}>0$ (independent of $\varepsilon$ ) such that for any $0<$ $\varepsilon<\min \left\{\bar{\varepsilon}_{0}, r_{0} / 2\right\}$,

$$
\begin{array}{r}
\sup _{0 \leq t \leq t_{0}} I_{\lambda, \mu}\left(t \bar{z}_{\varepsilon}^{i}\right)<c^{*}, \quad \text { uniformly in } i, \\
\forall 0<\lambda^{2 /(2-q)}+\mu^{2 /(2-q)}<\delta_{1} .
\end{array}
$$

According to condition (H1), $f_{\min }=\min _{x \in \bar{\Omega}} f(x)>0$ and $g_{\text {min }}=\min _{x \in \bar{\Omega}} g(x)>0$. Applying the results of Step I and (68), we have that for $N /(N-2)<q<2$ and $N>4$,

$$
\begin{aligned}
\sup _{t \geq t_{0}} I_{\lambda, \mu}\left(t \bar{z}_{\varepsilon}^{i}\right) & \\
= & \sup _{t \geq t_{0}}\left(I_{0,0}\left(t \bar{z}_{\varepsilon}^{i}\right)-\frac{t^{q}}{q} K_{\lambda, \mu}\left(t \bar{z}_{\varepsilon}^{i}\right)\right) \\
\leq & \frac{1}{N}\left(S_{\alpha, \beta}\right)^{N / 2}+O\left(\varepsilon^{N-2}\right)-\frac{t_{0}^{q}}{q} m(\lambda+\mu) \\
& \times \int_{B_{r_{0} / 2}\left(a_{i}\right)}\left|u_{\varepsilon}^{i}\right|^{q} d x \\
\leq & \frac{1}{N}\left(S_{\alpha, \beta}\right)^{N / 2}+O\left(\varepsilon^{N-2}\right)-(\lambda+\mu) O_{1}\left(\varepsilon^{\theta}\right),
\end{aligned}
$$

where $m=\min \left\{\alpha^{q / 2} f_{\min }, \beta^{q / 2} g_{\min }\right\}$ and $\theta=N-((N-2) q) / 2$.

Therefore, we can choose $\lambda=O_{1}\left(\varepsilon^{\tau_{1}}\right)$ and $\mu=O_{1}\left(\varepsilon^{\tau_{2}}\right)$ such that

$$
\frac{2-q}{q} \theta<\tau_{1}, \quad \tau_{2}<(N-2)-\theta
$$

This implies that

$$
\begin{gathered}
\min \left\{\tau_{1}, \tau_{2}\right\}+\theta<\frac{2}{2-q} \min \left(\tau_{1}, \tau_{2}\right), \\
\min \left\{\tau_{1}, \tau_{2}\right\}+\theta<N-2, \\
(\lambda+\mu) O_{1}\left(\varepsilon^{\theta}\right)=O_{1}\left(\varepsilon^{\min \left\{\tau_{1}, \tau_{2}\right\}+\theta}\right), \\
\lambda^{2 /(2-q)}+\mu^{2 /(2-q)}=O_{1}\left(\varepsilon^{2 /(2-q) \min \left\{\tau_{1}, \tau_{2}\right\}}\right) .
\end{gathered}
$$

There exist $\delta_{2}>0, \varepsilon_{0} \in\left(0, \min \left\{\bar{\varepsilon}_{0}, r_{0} / 2\right\}\right)$ such that for all $0<\lambda^{2 /(2-q)}+\mu^{2 /(2-q)}<\delta_{2}$ and $0<\varepsilon<\varepsilon_{0}$, we have

$$
O\left(\varepsilon^{N-2}\right)-(\lambda+\mu) O_{1}\left(\varepsilon^{\theta}\right)<-C_{0}\left(\lambda^{2 /(2-q)}+\mu^{2 /(2-q)}\right) .
$$

Thus, we can choose $\Lambda_{2}=\min \left\{(q / 2)^{2 /(2-q)} \Lambda_{1}, \delta_{1}, \delta_{2}\right\}>0$. Then, for all $\lambda^{2 /(2-q)}+\mu^{2 /(2-q)} \in\left(0, \Lambda_{2}\right)$, there holds

$$
\sup _{t \geq 0} I_{\lambda, \mu}\left(t \bar{z}_{\varepsilon}^{i}\right)<c^{*} \quad \text { uniformly in } i .
$$

Step III. For $0<\lambda^{2 /(2-q)}+\mu^{2 /(2-q)}<\Lambda_{2}$ and $0<\varepsilon<\varepsilon_{0}$, by Lemma 7, (72), and (85), we get

$$
0<\theta_{\lambda, \mu}^{-} \leq \theta_{\lambda, \mu}^{i} \leq I_{\lambda, \mu}\left(t \bar{z}_{\varepsilon}^{i}\right)<c^{*} \quad \forall 1 \leq i \leq k .
$$

To proceed, we need to quote the concentrationcompactness principle (see $[24,25]$ ) about the case of systems.

Lemma 15. Let $\left\{u_{n}, v_{n}\right\} \subset H_{0}^{1}(\Omega) \times H_{0}^{1}(\Omega)$ be a sequence such that

$$
\begin{gathered}
u_{n} \rightarrow u, \quad v_{n} \rightarrow v \quad \text { weakly in } H_{0}^{1}(\Omega) ; \\
u_{n} \longrightarrow u, \quad v_{n} \longrightarrow v \quad \text { a.e. on } \Omega,
\end{gathered}
$$$$
\left|\nabla\left(u_{n}-u\right)\right|^{2}+\left|\nabla\left(v_{n}-v\right)\right|^{2} \rightarrow \tilde{\mu}
$$$$
\text { weakly in the sense of measures, }
$$

$$
\left|u_{n}-u\right|^{\alpha}\left|v_{n}-v\right|^{\beta} \rightarrow \widetilde{v}
$$

weakly in the sense of measures.

Then, it follows that

$$
\begin{gathered}
\limsup _{n \rightarrow \infty} \int_{\mathbb{R}^{N}}\left(\left|\nabla u_{n}\right|^{2}+|\nabla v|_{n}^{2}\right) d x \\
=\int_{\mathbb{R}^{N}}\left(|\nabla u|^{2}+|\nabla v|^{2}\right) d x+\|\tilde{\mu}\|, \\
\limsup _{n \rightarrow \infty} \int_{\mathbb{R}^{N}}\left|u_{n}\right|^{\alpha}\left|v_{n}\right|^{\beta} d x=\int_{\mathbb{R}^{N}}|u|^{\alpha}|v|^{\beta} d x+\|\widetilde{\nu}\|, \\
\|\widetilde{\nu}\|^{2 /(\alpha+\beta)} \leq S_{\alpha, \beta}^{-1}\|\tilde{\mu}\| .
\end{gathered}
$$

Moreover, if $u \equiv v \equiv 0$ and $\|\widetilde{\nu}\|^{2 /(\alpha+\beta)}=S_{\alpha, \beta}^{-1}\|\widetilde{\mu}\|$, then $\widetilde{\mu}$ and $\widetilde{\nu}$ concentrate at a single point. 
Proof. See Han [20, Lemma 2.2].

Lemma 16. For any $i \in\{1,2, \ldots, k\}$, there exist $\widetilde{\Lambda}_{i}>0$ such that

$$
\widetilde{\theta}_{\lambda, \mu}^{i}>\frac{1}{N}\left(S_{\alpha, \beta}\right)^{N / 2} \quad \forall 0<\lambda^{2 /(2-q)}+\mu^{2 /(2-q)}<\widetilde{\Lambda}_{i} .
$$

Proof. Fix $i \in\{1,2, \ldots, k\}$. Assume the contrary. There then exists a sequence $\left\{\left(\lambda_{n}, \mu_{n}\right)\right\}$ with $\left(\lambda_{n}, \mu_{n}\right) \rightarrow(0,0)$ as $n \rightarrow$ $\infty$ such that $\widetilde{\theta}_{\lambda_{n}, \mu_{n}}^{i} \rightarrow c \leq(1 / N)\left(S_{\alpha, \beta}\right)^{N / 2}$ as $n \rightarrow \infty$. Consequently, there exists a sequence $\left\{z_{n}=\left(u_{n}, v_{n}\right)\right\} \subset$ $\partial \mathcal{N}_{\lambda_{n}, \mu_{n}}^{i}$ such that as $n \rightarrow \infty$,

$$
\begin{aligned}
& \int_{\Omega}\left(\left|\nabla u_{n}\right|^{2}+\left|\nabla v_{n}\right|^{2}\right) d x \\
& \quad=\int_{\Omega}\left(\lambda_{n} f(x)\left|u_{n}\right|^{q}+\mu_{n} g(x)\left|v_{n}\right|^{q}\right) d x \\
& \quad+\int_{\Omega} h(x)\left|u_{n}\right|^{\alpha}\left|v_{n}\right|^{\beta} d x, \\
& I_{\lambda_{n}, \mu_{n}}\left(z_{n}\right) \longrightarrow c \leq \frac{1}{N}\left(S_{\alpha, \beta}\right)^{N / 2} \text { as } n \longrightarrow \infty .
\end{aligned}
$$

It then follows easily that $\left\{z_{n}\right\}$ is uniformly bounded in $E$, and since $f$ and $g$ are continuous on $\bar{\Omega}$, we obtain

$$
\begin{aligned}
K_{\lambda_{n}, \mu_{n}}\left(z_{n}\right) & =\int_{\Omega}\left(\lambda_{n} f(x)\left|u_{n}\right|^{q}+\mu_{n} g(x)\left|v_{n}\right|^{q}\right) d x \\
& =o_{n}(1) \quad \text { as } n \longrightarrow \infty .
\end{aligned}
$$

From (90), and by the Hölder and the Sobolev inequalities, we can fix $m_{0}>0$ such that

$$
\begin{gathered}
\int_{\Omega}\left(\left|\nabla u_{n}\right|^{2}+\left|\nabla v_{n}\right|^{2}\right) d x \geq m_{0}, \\
\int_{\Omega} h(x)\left|u_{n}\right|^{\alpha}\left|v_{n}\right|^{\beta} d x \geq m_{0} .
\end{gathered}
$$

Thus, up to a subsequence, we infer that

$$
\begin{aligned}
\lim _{n \rightarrow \infty} & \int_{\Omega}\left(\left|\nabla u_{n}\right|^{2}+\left|\nabla v_{n}\right|^{2}\right) d x \\
& =\lim _{n \rightarrow \infty} \int_{\Omega} h(x)\left|u_{n}\right|^{\alpha}\left|v_{n}\right|^{\beta} d x=l>0 .
\end{aligned}
$$

Furthermore, by $|h|_{L^{\infty}(\Omega)}=1$, we deduce

$$
\begin{aligned}
l & =\lim _{n \rightarrow \infty} \int_{\Omega} h(x)\left|u_{n}\right|^{\alpha}\left|v_{n}\right|^{\beta} d x \\
& \leq|h|_{L^{\infty}(\Omega)} \lim _{n \rightarrow \infty} \int_{\Omega}\left|u_{n}\right|^{\alpha}\left|v_{n}\right|^{\beta} d x \\
& \leq S_{\alpha, \beta}^{-2^{*} / 2} \lim _{n \rightarrow \infty}\left(\int_{\Omega}\left(\left|\nabla u_{n}\right|^{2}+\left|\nabla v_{n}\right|^{2}\right) d x\right)^{2^{*} / 2} \\
& \leq S_{\alpha, \beta}^{-2^{*} / 2} l^{2^{*} / 2},
\end{aligned}
$$

which implies

$$
l \geq\left(S_{\alpha, \beta}\right)^{N / 2} .
$$

On the other hand, we have, as $n \rightarrow \infty$,

$$
\begin{aligned}
\frac{1}{N} l= & \frac{1}{2}\left\|z_{n}\right\|^{2} d x-\frac{1}{2^{*}} \int_{\Omega} h(x)\left|u_{n}\right|^{\alpha}\left|v_{n}\right|^{\beta} d x \\
& -\frac{1}{q} K_{\lambda_{n}, \mu_{n}}\left(z_{n}\right)+o_{n}(1) \\
= & I_{\lambda_{n}, \mu_{n}}\left(z_{n}\right)+o_{n}(1) \\
\leq & \frac{1}{N}\left(S_{\alpha, \beta}\right)^{N / 2} .
\end{aligned}
$$

Hence, together with (96), we get

$$
l=\left(S_{\alpha, \beta}\right)^{N / 2},
$$

and then from (95), we also have

$$
\lim _{n \rightarrow \infty} \int_{\Omega} h(x)\left|u_{n}\right|^{\alpha}\left|v_{n}\right|^{\beta} d x=\lim _{n \rightarrow \infty} \int_{\Omega}\left|u_{n}\right|^{\alpha}\left|v_{n}\right|^{\beta} d x=l .
$$

Therefore,

$$
\lim _{n \rightarrow \infty} \int_{\Omega}\left|u_{n}\right|^{\alpha}\left|v_{n}\right|^{\beta} d x=l .
$$

Set $\widetilde{z}_{n}=\left(\widetilde{u}_{n}, \widetilde{v}_{n}\right)=z_{n} /\left\|z_{n}\right\| ;$ then, we have $\left\|\widetilde{z}_{n}\right\|=1$. Moreover, by (94),(98), and (100), we get

$$
\lim _{n \rightarrow \infty} \int_{\Omega}\left|\widetilde{u}_{n}\right|^{\alpha}\left|\widetilde{v}_{n}\right|^{\beta} d x=\lim _{n \rightarrow \infty} \frac{\int_{\Omega}\left|u_{n}\right|^{\alpha}\left|v_{n}\right|^{\beta} d x}{\left\|z_{n}\right\|^{2^{*}}}=S_{\alpha, \beta}^{-N /(N-2)} .
$$

Thus, up to a subsequence, we may assume that

$$
\begin{gathered}
\tilde{u}_{n} \rightarrow u, \quad \tilde{v}_{n} \rightarrow v \quad \text { weakly in } H_{0}^{1}(\Omega) ; \\
\widetilde{u}_{n} \longrightarrow u, \quad \widetilde{v}_{n} \longrightarrow v \quad \text { a.e. on } \Omega, \\
\left|\nabla\left(\widetilde{u}_{n}-u\right)\right|^{2}+\left|\nabla\left(\widetilde{v}_{n}-v\right)\right|^{2} \rightarrow \tilde{\mu}
\end{gathered}
$$

weakly in the sense of measures,

$$
\left|\widetilde{u}_{n}-u\right|^{\alpha}\left|\widetilde{v}_{n}-v\right|^{\beta}-\widetilde{v}
$$

weakly in the sense of measures.

Since $\Omega$ is bounded, from (101) and Lemma 15 , we deduce that

$$
\begin{gathered}
1=\int_{\Omega}\left(|\nabla u|^{2}+|\nabla v|^{2}\right) d x+\|\widetilde{\mu}\|, \\
S_{\alpha, \beta}^{-N /(N-2)}=\int_{\Omega}|u|^{\alpha}|v|^{\beta} d x+\|\tilde{\nu}\|, \\
\|\widetilde{\nu}\|^{2 /(\alpha+\beta)} \leq S_{\alpha, \beta}^{-1}\|\widetilde{\mu}\| .
\end{gathered}
$$


If $\int_{\Omega}\left(|\nabla u|^{2}+|\nabla v|^{2}\right) d x \neq 0$ and $\|\widetilde{\mu}\| \neq 0$, we deduce that

$$
\begin{aligned}
1 & =\left(\int_{\Omega}\left(|\nabla u|^{2}+|\nabla v|^{2}\right) d x+\|\widetilde{\mu}\|\right)^{(\alpha+\beta) / 2} \\
& >\left(\int_{\Omega}\left(|\nabla u|^{2}+|\nabla v|^{2}\right) d x\right)^{(\alpha+\beta) / 2}+\|\widetilde{\mu}\|^{(\alpha+\beta) / 2} \\
& \geq S_{\alpha, \beta}^{(\alpha+\beta) / 2} \int_{\Omega}|u|^{\alpha}|v|^{\beta} d x+S_{\alpha, \beta}^{(\alpha+\beta) / 2}\|\widetilde{\nu}\| \\
& =S_{\alpha, \beta}^{(\alpha+\beta) / 2} \cdot S_{\alpha, \beta}^{-N /(N-2)} \\
& =1
\end{aligned}
$$

which is a contradiction.

Thus, $\int_{\Omega}\left(|\nabla u|^{2}+|\nabla v|^{2}\right) d x=0$ or $\|\widetilde{\mu}\|=0$. If $\|\widetilde{\mu}\|=$ 0 , from (103)-(105), we get $\int_{\Omega}\left(|\nabla u|^{2}+|\nabla v|^{2}\right) d x=1$ and $\int_{\Omega}|u|^{\alpha}|v|^{\beta} d x=S_{\alpha, \beta}^{-N /(N-2)}$. Then,

$$
\frac{\int_{\Omega}\left(|\nabla u|^{2}+|\nabla v|^{2}\right) d x}{\left(\int_{\Omega}|u|^{\alpha}|v|^{\beta} d x\right)^{2 /(\alpha+\beta)}}=S_{\alpha, \beta},
$$

which means that $S_{\alpha, \beta}$ is achieved by $(u, v)$. It is impossible since $S_{\alpha, \beta}$ cannot be achieved on any bounded domain $\Omega$. Hence,

$$
\int_{\Omega}\left(|\nabla u|^{2}+|\nabla v|^{2}\right) d x=0, \quad\|\widetilde{\mu}\|=1 .
$$

Then, $u \equiv v \equiv 0$ on $\Omega$, and from (103), (104), we easily have $\|\widetilde{\nu}\|^{2 /(\alpha+\beta)}=S_{\alpha, \beta}^{-1}=S_{\alpha, \beta}^{-1}\|\widetilde{\mu}\|$. By Lemma 15, we conclude that $x_{0} \in \bar{\Omega}$ such that

$$
\left|\nabla \widetilde{u}_{n}\right|^{2}+|\nabla \widetilde{v}|^{2}-\delta_{x_{0}}
$$

weakly in the sense of measures,

$$
\left|\widetilde{u}_{n}\right|^{\alpha}\left|\widetilde{v}_{n}\right|^{\beta} \rightarrow S_{\alpha, \beta}^{-N /(N-2)} \delta_{x_{0}}
$$

weakly in the sense of measures.

Observe that $Q_{i}\left(\widetilde{z}_{n}\right)=Q_{i}\left(z_{n}\right)=r_{0} / 3$;

$$
\begin{aligned}
\frac{r_{0}}{3} & =\lim _{n \rightarrow \infty} Q_{i}\left(z_{n}\right) \\
& =\lim _{n \rightarrow \infty} \frac{\int_{\Omega} \psi_{i}(x)\left(\left|\nabla \widetilde{u}_{n}\right|^{2}+\left|\nabla \widetilde{v}_{n}\right|^{2}\right) d x}{\int_{\Omega}\left(\left|\nabla \widetilde{u}_{n}\right|^{2}+\left|\nabla \widetilde{v}_{n}\right|^{2}\right) d x}=\psi_{i}\left(x_{0}\right),
\end{aligned}
$$

which implies that $x_{0} \neq a_{i}$ by the definition of $\psi_{i}(x)$. On the other hand, from (95) and (101), we get

$$
\begin{aligned}
S_{\alpha, \beta}^{-N /(N-2)} h\left(x_{0}\right) & =\lim _{n \rightarrow \infty} \int_{\Omega} h(x)\left|\tilde{u}_{n}\right|^{\alpha}\left|\tilde{v}_{n}\right|^{\beta} d x \\
& =\lim _{n \rightarrow \infty} \frac{\int_{\Omega} h(x)\left|u_{n}\right|^{\alpha}\left|v_{n}\right|^{\beta} d x}{\left\|z_{n}\right\|^{2^{*}}} \\
& =\lim _{n \rightarrow \infty} \frac{\int_{\Omega}\left|u_{n}\right|^{\alpha}\left|v_{n}\right|^{\beta} d x}{\left\|z_{n}\right\|^{2^{*}}} \\
& =\lim _{n \rightarrow \infty} \int_{\Omega}\left|\widetilde{u}_{n}\right|^{\alpha}\left|\widetilde{v}_{n}\right|^{\beta} d x \\
& =S_{\alpha, \beta}^{-N /(N-2)},
\end{aligned}
$$

which is impossible, because $h(x)$ is not a constant function by condition $(\mathrm{H} 2)$.

Throughout this section, take $\Lambda^{*}=\min \left\{\Lambda_{2}, \min _{1 \leq i \leq k} \widetilde{\Lambda}_{i}\right\} ;$ $\Lambda_{2}$ and $\widetilde{\Lambda}_{i}$ are as in Lemmas 14 and 16. Using the idea of Tarantello [12], we have the following results. For $z=(u, v)$, $\varphi=\left(\varphi_{1}, \varphi_{2}\right) \in E$, we define

$$
\begin{gathered}
z-\varphi=\left(u-\varphi_{1}, v-\varphi_{2}\right), \\
\langle z, \varphi\rangle=\int_{\Omega}\left(\nabla u \nabla \varphi_{1}+\nabla v \nabla \varphi_{2}\right) d x,
\end{gathered}
$$

$$
\begin{aligned}
G_{\lambda, \mu}(z, \varphi)=\int_{\Omega}\left(\lambda f(x)|u|^{q-2} u \varphi_{1}+\mu g(x)|v|^{q-2} v \varphi_{2}\right) d x & \\
H(z, \varphi)= & \frac{\alpha}{\alpha+\beta} \int_{\Omega} h(x)|u|^{\alpha-2} u|v|^{\beta} \varphi_{1} d x \\
& +\frac{\beta}{\alpha+\beta} \int_{\Omega} h(x)|u|^{\alpha}|v|^{\beta-2} v \varphi_{2} d x .
\end{aligned}
$$

Lemma 17. For each $0<\lambda^{2 /(2-q)}+\mu^{2 /(2-q)}<\Lambda^{*}$ and $z=$ $(u, v) \in \mathcal{N}_{\lambda, \mu}^{i}(1 \leq i \leq k)$, there exist $\epsilon>0$ and a differentiable function $\xi: B_{\epsilon}(0) \subset E \rightarrow \mathbb{R}^{+}$such that $\xi(0)=1, \xi(\phi)(z-\phi) \epsilon$ $\mathcal{N}_{\lambda, \mu}^{i}$ for all $\phi \in B_{\epsilon}(0)$ and

$$
\left\langle\xi^{\prime}(0), \varphi\right\rangle=\frac{2\langle z, \varphi\rangle-q G_{\lambda, \mu}(z, \varphi)-2^{*} H(z, \varphi)}{(2-q)\|z\|_{E}^{2}-\left(2^{*}-q\right) H(z, z)},
$$

for all $\varphi=\left(\varphi_{1}, \varphi_{2}\right) \in E$.

Proof. For $z \in \mathcal{N}_{\lambda, \mu}^{i}$, define a function $F_{z}: \mathbb{R} \times E \rightarrow \mathbb{R}$ by

$$
\begin{aligned}
F_{z}(\xi, \phi)= & \left\langle I_{\lambda, \mu}^{\prime}(\xi(z-\phi)), \xi(z-\phi)\right\rangle \\
= & \xi^{2}\|z-\phi\|^{2}-\xi^{q} G_{\lambda, \mu}(z-\phi, z-\phi) \\
& -\xi^{\alpha+\beta} H(z-\phi, z-\phi) .
\end{aligned}
$$

Then, $F_{u}(1,0)=\left\langle I_{\lambda, \mu}^{\prime}(z), z\right\rangle=0$ and

$$
\begin{aligned}
\frac{d}{d \xi} F_{z}(1,0) & =2\|z\|_{E}^{2}-q G_{\lambda, \mu}(z, z)-(\alpha+\beta) H(z, z) \\
& =(2-q)\|z\|_{E}^{2}-\left(2^{*}-q\right) H(z, z)<0 .
\end{aligned}
$$


According to the implicit function theorem, there exist $\epsilon>0$ and a differentiable function $\xi: B_{\epsilon}(0) \subset E \rightarrow \mathbb{R}$ such that $\xi(0)=1$;

$$
\begin{gathered}
\left\langle\xi^{\prime}(0), \varphi\right\rangle=\frac{2\langle z, \varphi\rangle-q G_{\lambda, \mu}(z, \varphi)-2^{*} H(z, \varphi)}{(2-q)\|z\|_{E}^{2}-\left(2^{*}-q\right) H(z, z)}, \\
F_{z}(\xi(\varphi), \varphi)=0 \quad \forall \varphi \in B_{\epsilon}(0),
\end{gathered}
$$

which is equivalent to

$$
\left\langle I_{\lambda, \mu}^{\prime}(\xi(\varphi)(z-\varphi)), \xi(\varphi)(z-\varphi)\right\rangle=0 \quad \forall \varphi \in B(0 ; \epsilon) ;
$$

that is, $\xi(\varphi)(z-\varphi) \in \mathcal{N}_{\lambda, \mu}$ for all $\varphi \in B_{\epsilon}(0)$. Furthermore, by the continuity of the functions $\xi$ and $Q_{i}$, we have that

$$
\begin{gathered}
(2-q)\|\xi(\varphi)(z-\varphi)\|^{2} \\
-\left(2^{*}-q\right) H(\xi(\varphi)(z-\varphi), \xi(\varphi)(z-\varphi))<0, \\
Q_{i}(\xi(\varphi)(z-\varphi))<\frac{r_{0}}{3}
\end{gathered}
$$

still holds if $\epsilon$ is sufficiently small. This implies that $\xi(\varphi)(z-$ $\varphi) \in \mathcal{N}_{\lambda, \mu}^{i}$.

Proposition 18. If $0<\lambda^{2 /(2-q)}+\mu^{2 /(2-q)}<\Lambda^{*}$, then there

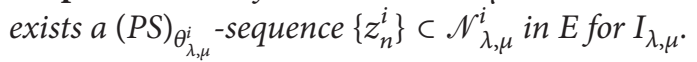

Proof. If $\overline{\mathcal{N}_{\lambda, \mu}^{i}}$ denotes the closure of $\mathcal{N}_{\lambda, \mu}^{i}$, at first we note that $\overline{\mathcal{N}_{\lambda, \mu}^{i}}=\mathcal{N}_{\lambda, \mu}^{i} \cup \partial \mathcal{N}_{\lambda, \mu}^{i}$ for all $i=1,2, \ldots, k$. It then follows from Lemmas 14 and 16, that

$\theta_{\lambda, \mu}^{i}<\widetilde{\theta}_{\lambda, \mu}^{i} \quad$ for $i=1,2, \ldots, k, 0<\lambda^{2 /(2-q)}+\mu^{2 /(2-q)}<\Lambda^{*}$.

Hence,

$$
\theta_{\lambda, \mu}^{i}=\inf \left\{I_{\lambda, \mu}(z) \mid z \in \overline{\mathcal{N}_{\lambda, \mu}^{i}}\right\} \quad \text { for } i=1,2, \ldots, k .
$$

Now, we fix $i \in\{1,2, \ldots, k\}$. Applying the Ekeland variational principle [26], there exists a minimizing sequence $\left\{z_{n}^{i}\right\} \subset$ $\overline{\mathcal{N}_{\lambda, \mu}^{i}}$ such that

$$
\begin{gathered}
I_{\lambda, \mu}\left(z_{n}^{i}\right)<\theta_{\lambda, \mu}^{i}+\frac{1}{n} \\
I_{\lambda, \mu}\left(z_{n}^{i}\right) \leq I_{\lambda, \mu}(\varphi)+\frac{1}{n}\left\|\varphi-z_{n}^{i}\right\|_{E} \quad \text { for each } \varphi \in \overline{\mathcal{N}_{\lambda, \mu}^{i}} .
\end{gathered}
$$

Using (119), we may assume that $z_{n}^{i} \in \mathcal{N}_{\lambda, \mu}^{i}$ for $n$ sufficiently large. Applying Lemma 17 with $z=z_{n}^{i}$, we obtain the function $\xi_{n}: B_{\epsilon_{n}}(0) \rightarrow \mathbb{R}$ for some $\epsilon_{n}>0$ such that $\xi_{n}(\varphi)\left(z_{n}^{i}-\varphi\right) \epsilon$ $\mathcal{N}_{\lambda, \mu}^{i}$ for all $\varphi \in B_{\epsilon_{n}}(0)$. Let $0<\delta<\epsilon_{n}$ and $z \in E \backslash\{0\}$; we set

$$
\varphi_{\delta}=\frac{\delta z}{\|z\|_{E}}
$$

and $z_{\delta}=\xi_{n}\left(\varphi_{\delta}\right)\left(z_{n}^{i}-\varphi_{\delta}\right)$. Since $z_{\delta} \in \mathcal{N}_{\lambda, \mu}^{i}$, we deduce from (121) that

$$
I_{\lambda, \mu}\left(z_{\delta}\right)-I_{\lambda, \mu}\left(z_{n}^{i}\right) \geq-\frac{1}{n}\left\|z_{\delta}-z_{n}^{i}\right\|_{E}
$$

By the mean-value theorem, we obtain

$$
\left\langle I_{\lambda, \mu}^{\prime}\left(z_{n}^{i}\right),\left(z_{\delta}-z_{n}^{i}\right)\right\rangle+o\left(\left\|z_{\delta}-z_{n}^{i}\right\|_{E}\right) \geq-\frac{1}{n}\left\|z_{\delta}-z_{n}^{i}\right\|_{E} .
$$

Therefore,

$$
\begin{gathered}
\left\langle I_{\lambda, \mu}^{\prime}\left(z_{n}^{i}\right),-\varphi_{\delta}\right\rangle+\left(\xi_{n}\left(\varphi_{\delta}\right)-1\right)\left\langle I_{\lambda, \mu}^{\prime}\left(z_{n}^{i}\right),\left(z_{n}^{i}-\varphi_{\delta}\right)\right\rangle \\
\geq-\frac{1}{n}\left\|z_{\delta}-z_{n}^{i}\right\|_{E}+o\left(\left\|z_{\delta}-z_{n}^{i}\right\|_{E}\right) .
\end{gathered}
$$

Now, we observe that $\xi_{n}\left(\varphi_{\delta}\right)\left(z_{n}^{i}-\varphi_{\delta}\right) \in \mathcal{N}_{\lambda, \mu}^{i}$, and consequently we get from (125) that

$$
\begin{aligned}
&-\delta\left\langle I_{\lambda, \mu}^{\prime}\left(z_{n}^{i}\right), \frac{z}{\|z\|_{E}}\right\rangle \\
& \quad+\frac{\left(\xi_{n}\left(\varphi_{\delta}\right)-1\right)}{\xi_{n}\left(\varphi_{\delta}\right)}\left\langle I_{\lambda, \mu}^{\prime}\left(z_{\delta}\right), \xi_{n}\left(\varphi_{\delta}\right)\left(z_{n}^{i}-\varphi_{\delta}\right)\right\rangle \\
& \quad+\left(\xi_{n}\left(\varphi_{\delta}\right)-1\right)\left\langle I_{\lambda, \mu}^{\prime}\left(z_{n}^{i}\right)-I_{\lambda, \mu}^{\prime}\left(z_{\delta}\right),\left(z_{n}^{i}-\varphi_{\delta}\right)\right\rangle \\
& \geq-\frac{1}{n}\left\|z_{\delta}-z_{n}^{i}\right\|_{E}+o\left(\left\|z_{\delta}-z_{n}^{i}\right\|_{E}\right) .
\end{aligned}
$$

Then, we write the pervious inequality in the following form:

$$
\begin{aligned}
\left\langle I_{\lambda, \mu}^{\prime}\left(z_{n}^{i}\right), \frac{z}{\|z\|_{E}}\right\rangle & \\
\leq & \frac{\left\|z_{\delta}-z_{n}^{i}\right\|_{E}}{\delta n}+\frac{o\left(\left\|z_{\delta}-z_{n}^{i}\right\|_{E}\right)}{\delta} \\
& +\frac{\left(\xi_{n}\left(\varphi_{\delta}\right)-1\right)}{\delta}\left\langle I_{\lambda, \mu}^{\prime}\left(z_{n}^{i}\right)-I_{\lambda, \mu}^{\prime}\left(z_{\delta}\right),\left(z_{n}^{i}-\varphi_{\delta}\right)\right\rangle .
\end{aligned}
$$

We can find a constant $C>0$ independent of $\delta$ such that

$$
\begin{gathered}
\left\|z_{\delta}-z_{n}^{i}\right\| \leq \delta+C\left(\left|\xi_{n}\left(\varphi_{\delta}\right)-1\right|\right), \\
\lim _{\delta \rightarrow 0} \frac{\left|\xi_{n}\left(\varphi_{\delta}\right)-1\right|}{\delta} \leq\left\|\xi_{n}^{\prime}(0)\right\| \leq C .
\end{gathered}
$$

For a fixed $n$, let $\delta \rightarrow 0$ in (127). Using the fact that

$$
\lim _{\delta \rightarrow 0}\left\|z_{\delta}-z_{n}^{i}\right\|_{E}=0
$$

we obtain

$$
\left\langle I_{\lambda, \mu}^{\prime}\left(z_{n}^{i}\right), \frac{z}{\|z\|_{E}}\right\rangle \leq \frac{C}{n} .
$$


This implies

$$
I_{\lambda, \mu}\left(z_{n}^{i}\right)=\theta_{\lambda, \mu}^{i}+o_{n}(1), \quad I_{\lambda, \mu}^{\prime}\left(z_{n}^{i}\right)=o_{n}(1) \quad \text { in } E^{-1} .
$$

Now, we complete the proof of Theorem 2. By Lemmas 12, 14 and Proposition 18, for all $\lambda^{2 /(2-q)}+\mu^{2 /(2-q)} \in\left(0, \Lambda^{*}\right)$, there exists a sequence $\left\{z_{n}^{i}\right\} \subset \mathcal{N}_{\lambda, \mu}^{i}$ and $z_{0}^{i}=\left(u_{0}^{i}, v_{0}^{i}\right) \in E, 1 \leq i \leq k$, such that

$$
\begin{gathered}
I_{\lambda, \mu}\left(z_{n}^{i}\right)=\theta_{\lambda, \mu}^{i}+o_{n}(1), \\
I_{\lambda, \mu}^{\prime}\left(z_{n}^{i}\right)=o_{n}(1) \quad \text { in } E^{-1}, \\
z_{n}^{i} \longrightarrow z_{0}^{i} \quad \text { strongly in } E .
\end{gathered}
$$

Moreover, $\left\{z_{n}^{i}\right\} \subset \mathcal{N}_{\lambda, \mu}^{-}$, and by Lemma 7 (ii), we get $z_{0}^{i} \in$ $\mathcal{N}_{\lambda, \mu}^{-}, u_{0}^{i} \not \equiv 0, v_{0}^{i} \not \equiv 0$ in $\Omega$,

$$
\begin{gathered}
\left\|z_{0}^{i}\right\|_{E}>\left(\frac{2-q}{2^{*}-q}\right)^{1 /\left(2^{*}-2\right)} S^{N / 4}, \\
\theta_{\lambda, \mu}^{i} \geq \theta_{\lambda, \mu}^{-}>0 \quad \text { for } i=1,2, \ldots, k .
\end{gathered}
$$

Thus, $z_{0}^{i}$ is a nontrivial solution of the problem $\left(P_{\lambda, \mu}\right)$ and $I_{\lambda, \mu}\left(z_{0}^{i}\right)=\theta_{\lambda, \mu}^{i}$ for $i=1,2 \ldots, k$. Set $u_{+}=\max \{u, 0\}$ and $v_{+}=\max \{v, 0\}$. Replace the terms $\int_{\Omega} h(x)|u|^{\alpha}|v|^{\beta} d x$ and $\int_{\Omega}\left(\lambda f(x)|u|^{q}+\mu g(x)|v|^{q}\right) d x$ of the functional $I_{\lambda, \mu}$ by $\int_{\Omega} h(x) u_{+}^{\alpha} v_{+}^{\beta} d x$ and $\int_{\Omega}\left(\lambda f(x) u_{+}^{q}+\mu g(x) v_{+}^{q}\right) d x$, respectively. It then follows that $z_{0}^{i}$ is a nonnegative solution of the problem $\left(P_{\lambda, \mu}\right)$. Applying the maximum principle [19], $z_{0}^{i}$ is a positive solution of the problem $\left(P_{\lambda, \mu}\right)$. Since $Q_{i}\left(z_{0}^{i}\right)<r_{0} / 3$,

$$
z_{\lambda, \mu}^{1} \in \mathcal{N}_{\lambda, \mu}^{+}, \quad z_{0}^{i} \in \mathcal{N}_{\lambda, \mu}^{i} \subset \mathcal{N}_{\lambda, \mu}^{-} \text {for } i=1,2, \ldots, k,
$$

where $z_{\lambda, \mu}^{1}$ is a positive solution of equation $\left(P_{\lambda, \mu}\right)$ as in Theorem 1. From Lemma 13, we conclude that $\mathcal{N}_{\lambda, \mu}^{i}$ are disjoint for $i=1,2 \ldots, k$. This implies that $z_{0}^{i}(1 \leq i \leq k)$ and $z_{\lambda, \mu}^{1}$ are distinct positive solutions of the problem $\left(P_{\lambda, \mu}\right)$.

\section{References}

[1] C. O. Alves, D. C. de Morais Filho, and M. A. S. Souto, "On systems of elliptic equations involving subcritical or critical Sobolev exponents," Nonlinear Analysis: Theory, Methods \& Applications, vol. 42, no. 5, pp. 771-787, 2000.

[2] K. J. Brown and T.-F. Wu, "A semilinear elliptic system involving nonlinear boundary condition and sign-changing weight function," Journal of Mathematical Analysis and Applications, vol. 337, no. 2, pp. 1326-1336, 2008.

[3] D. Cao and P. Han, "High energy positive solutions of Neumann problem for an elliptic system of equations with critical nonlinearities," Calculus of Variations and Partial Differential Equations, vol. 25, no. 2, pp. 161-185, 2006.
[4] J. Chabrowski and J. Yang, "On the Neumann problem for an elliptic system of equations involving the critical Sobolev exponent," Colloquium Mathematicum, vol. 90, no. 1, pp. 19-35, 2001.

[5] C.-M. Chu and C.-L. Tang, "Existence and multiplicity of positive solutions for semilinear elliptic systems with Sobolev critical exponents," Nonlinear Analysis. Theory, Methods \& Applications, vol. 71, no. 11, pp. 5118-5130, 2009.

[6] P. Han, "Multiple positive solutions of nonhomogeneous elliptic systems involving critical Sobolev exponents," Nonlinear Analysis: Theory, Methods \& Applications, vol. 64, no. 4, pp. 869-886, 2006.

[7] T.-S. Hsu, "Multiple positive solutions for a critical quasilinear elliptic system with concave-convex nonlinearities," Nonlinear Analysis: Theory, Methods \& Applications, vol. 71, no. 7-8, pp. 2688-2698, 2009.

[8] T.-S. Hsu and H.-L. Lin, "Multiple positive solutions for a critical elliptic system with concave-convex nonlinearities," Proceedings of the Royal Society of Edinburgh. Section A, vol. 139, no. 6, pp. 1163-1177, 2009.

[9] Y. Shen and J. Zhang, "Multiplicity of positive solutions for a semilinear $p$-Laplacian system with Sobolev critical exponent," Nonlinear Analysis: Theory, Methods \& Applications, vol. 74, no. 4, pp. 1019-1030, 2011.

[10] T.-F. Wu, "The Nehari manifold for a semilinear elliptic system involving sign-changing weight functions," Nonlinear Analysis: Theory, Methods \& Applications, vol. 68, no. 6, pp. 1733-1745, 2008.

[11] H.-1. Lin, "Multiple positive solutions for semilinear elliptic systems," Journal of Mathematical Analysis and Applications, vol. 391, no. 1, pp. 107-118, 2012.

[12] G. Tarantello, "On nonhomogeneous elliptic equations involving critical Sobolev exponent," Annales de l'Institut Henri Poincaré. Analyse Non Linéaire, vol. 9, no. 3, pp. 281-304, 1992.

[13] G. Tarantello, "Multiplicity results for an inhomogeneous Neumann problem with critical exponent," Manuscripta Mathematica, vol. 81, no. 1-2, pp. 57-78, 1993.

[14] T.-S. Hsu, "Multiplicity results for $p$-Laplacian with critical nonlinearity of concave-convex type and sign-changing weight functions," Abstract and Applied Analysis, vol. 2009, Article ID 652109, 24 pages, 2009.

[15] K. J. Brown and Y. Zhang, "The Nehari manifold for a semilinear elliptic equation with a sign-changing weight function," Journal of Differential Equations, vol. 193, no. 2, pp. 481-499, 2003.

[16] T.-F. Wu, "On semilinear elliptic equations involving concaveconvex nonlinearities and sign-changing weight function," Journal of Mathematical Analysis and Applications, vol. 318, no. 1, pp. 253-270, 2006.

[17] H. Brézis and E. Lieb, "A relation between pointwise convergence of functions and convergence of functionals," Proceedings of the American Mathematical Society, vol. 88, no. 3, pp. 486490, 1983.

[18] T.-S. Hsu, "Existence and multiplicity of positive solutions to a perturbed singular elliptic system deriving from a strongly coupled critical potential," Boundary Value Problems, vol. 116, 14 pages, 2012.

[19] J. L. Vázquez, "A strong maximum principle for some quasilinear elliptic equations," Applied Mathematics and Optimization, vol. 12, no. 3, pp. 191-202, 1984.

[20] P. Han, "The effect of the domain topology on the number of positive solutions of an elliptic system involving critical Sobolev 
exponents," Houston Journal of Mathematics, vol. 32, no. 4, pp. 1241-1257, 2006.

[21] H. Brézis and L. Nirenberg, "Positive solutions of nonlinear elliptic equations involving critical Sobolev exponents," Communications on Pure and Applied Mathematics, vol. 36, no. 4, pp. 437-477, 1983.

[22] X. Cheng and S. Ma, "Existence of three nontrivial solutions for elliptic systems with critical exponents and weights," Nonlinear Analysis: Theory, Methods \& Applications, vol. 69, no. 10, pp. 3537-3548, 2008.

[23] M. Struwe, Variational Methods, vol. 34 of Results in Mathematics and Related Areas (3), Springer, Berlin, Germany, 2nd edition, 1996.

[24] M. Willem, Minimax Theorems, Progress in Nonlinear Differential Equations and Their Applications, 24, Birkhäuser, Boston, Mass, USA, 1996.

[25] G. Bianchi, J. Chabrowski, and A. Szulkin, "On symmetric solutions of an elliptic equation with a nonlinearity involving critical Sobolev exponent," Nonlinear Analysis: Theory, Methods \& Applications, vol. 25, no. 1, pp. 41-59, 1995.

[26] I. Ekeland, "On the variational principle," Journal of Mathematical Analysis and Applications, vol. 47, pp. 324-353, 1974. 


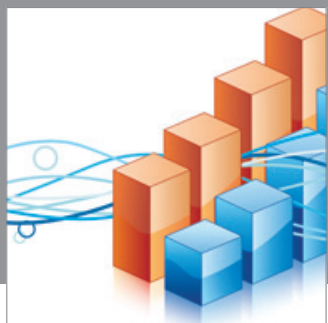

Advances in

Operations Research

mansans

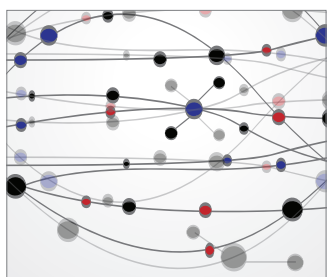

The Scientific World Journal
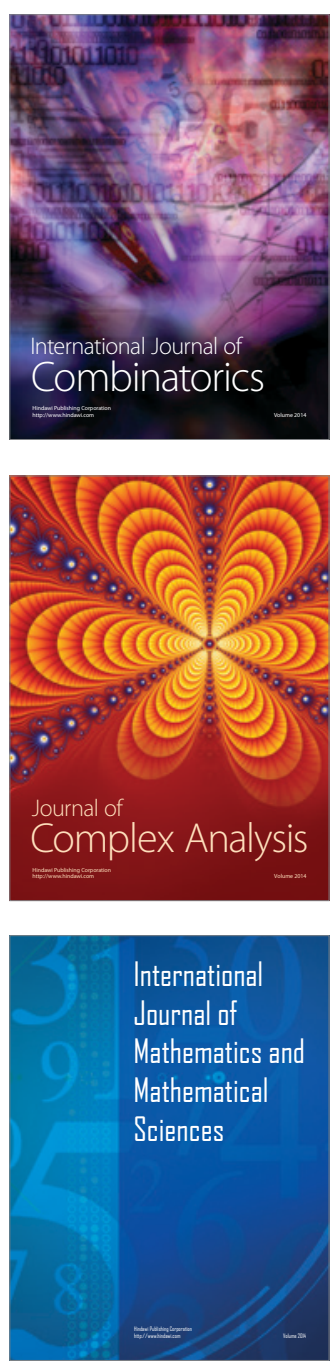
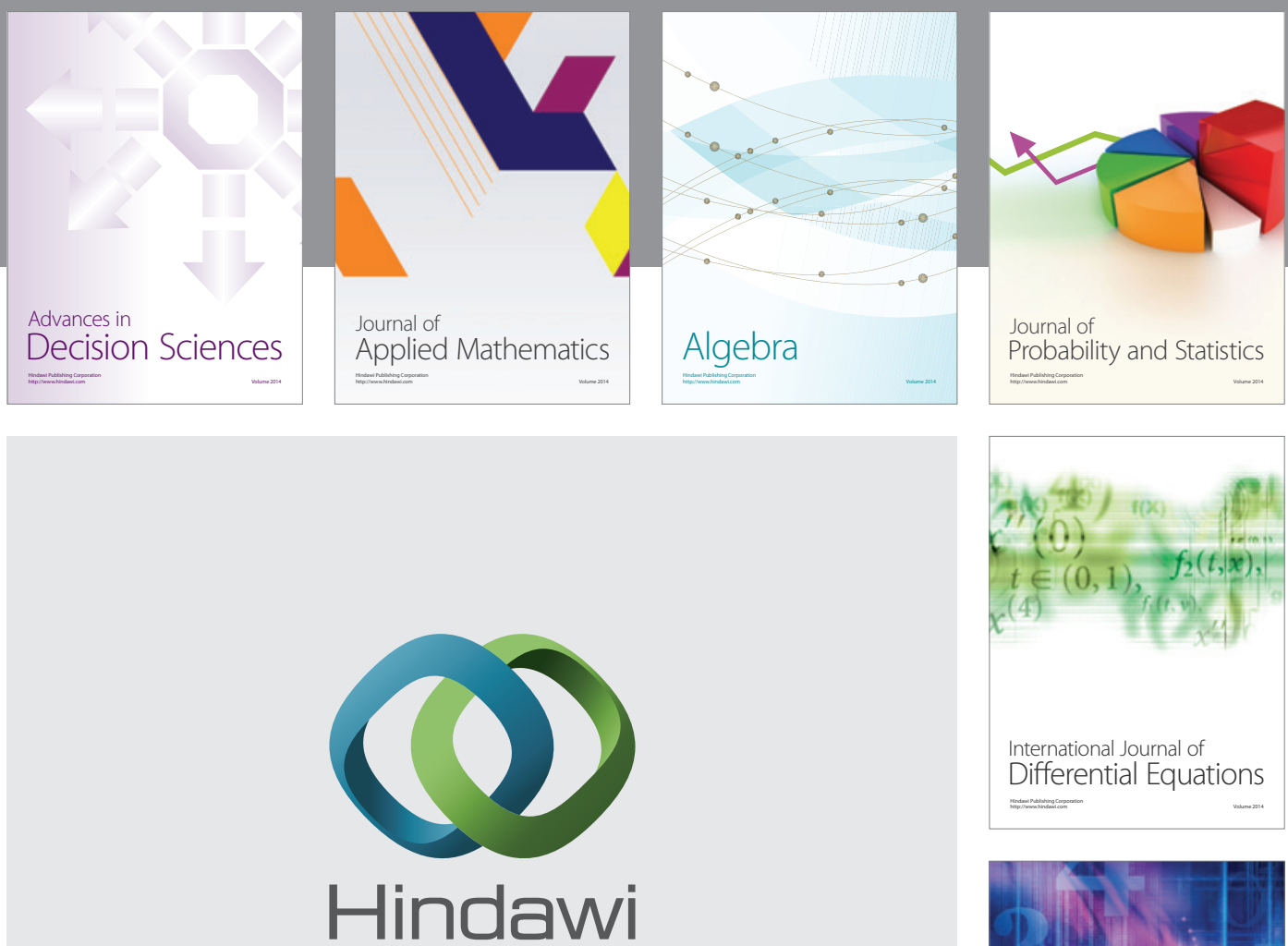

Submit your manuscripts at http://www.hindawi.com
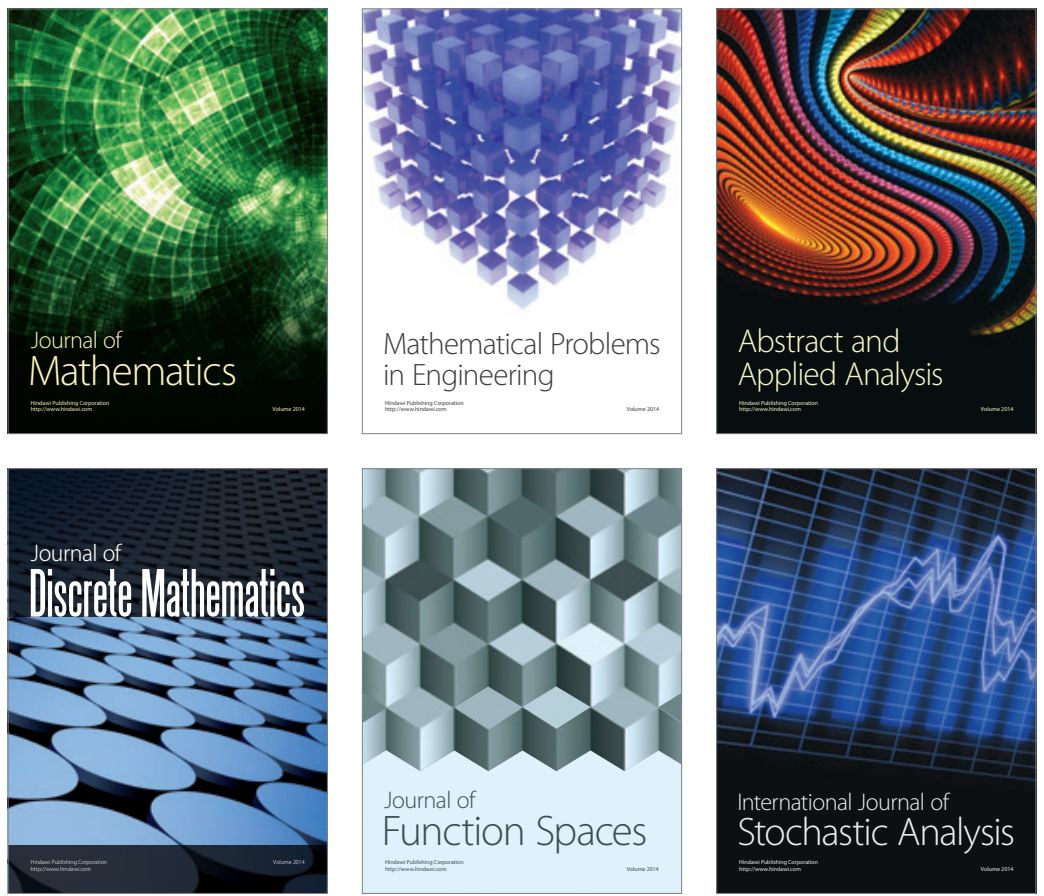

Journal of

Function Spaces

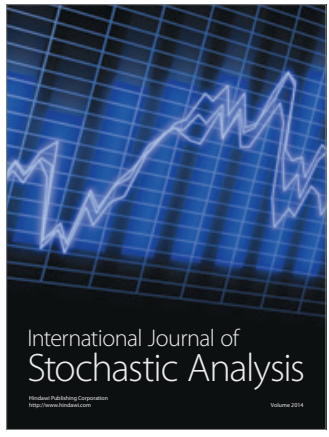

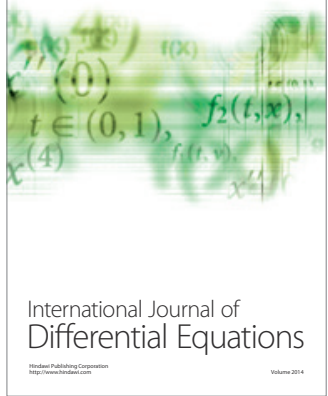
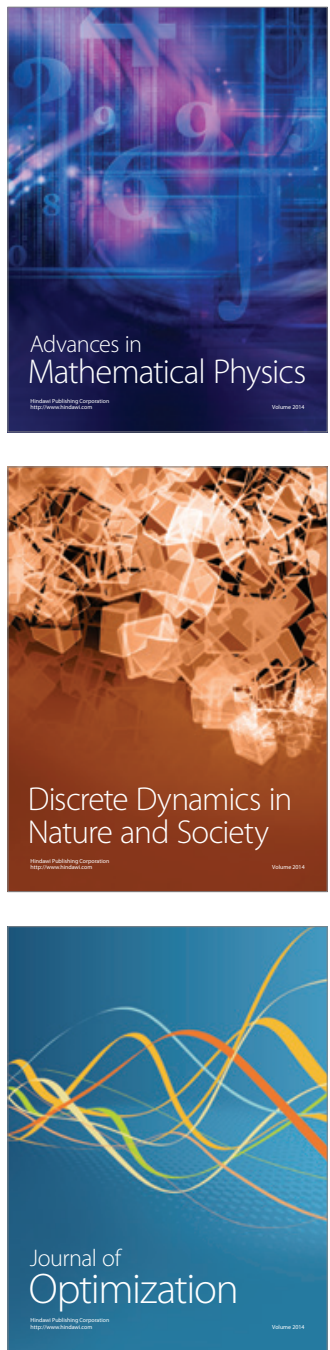